\title{
Computer simulations of glasses: the potential energy landscape
}

\author{
Zamaan Raza, Björn Alling and Igor Abrikosov
}

Linköping University Post Print

Tweet

N.B.: When citing this work, cite the original article.

Original Publication:

Zamaan Raza, Björn Alling and Igor Abrikosov, Computer simulations of glasses: the potential energy landscape, 2015, Journal of Physics: Condensed Matter, (27), 29, 293201.

http://dx.doi.org/10.1088/0953-8984/27/29/293201

Copyright: IOP Publishing: Hybrid Open Access

http://www.iop.org/

Postprint available at: Linköping University Electronic Press

http://urn.kb.se/resolve?urn=urn:nbn:se:liu:diva-120738 


\title{
Topical Review
}

\section{Computer simulations of glasses: the potential energy landscape}

\author{
Zamaan Raza $^{1}$, Björn Alling ${ }^{1}$ and Igor A. Abrikosov ${ }^{1,2,3}$ \\ ${ }^{1}$ Department of Physics, Biology and Chemistry (IFM), Linköping University, \\ SE-581 83, Linköping, Sweden \\ 2 Materials Modeling and Development Laboratory, NUST "MISIS", 119049 \\ Moscow, Russia \\ ${ }^{3}$ LOCOMAS Laboratory, Tomsk State University, 634050 Tomsk, Russia \\ E-mail: zamaan.raza@gmail.com
}

\begin{abstract}
We review the current state of research on glasses, discussing the theoretical background and computational models employed to describe them. This article focuses on the use of the potential energy landscape (PEL) paradigm to account for the phenomenology of glassy systems, and the way in which it can be applied in simulations and the interpretation of their results. This article provides a broad overview of the rich phenomenology of glasses, followed by a summary of the theoretical frameworks developed to describe this phenomonology. We discuss the background of the PEL in detail, the onerous task of how to generate computer models of glasses, various methods of analysing numerical simulations, and the literature on the most commonly used model systems. Finally, we tackle the problem of how to distinguish a good glass former from a good crystal former from an analysis of the PEL. In summarising the state of the potential energy landscape picture, we develop the foundations for new theoretical methods that allow the $a b$ initio prediction of the glass-forming ability of new materials by analysis of the PEL.

Keywords: Glasses, Potential Energy Landscape, Computer Simulation
\end{abstract}

PACS numbers: 61.43.-j,61.43.Fs,64.70.kj,64.70.Q-

Submitted to: J. Phys.: Condens. Matter 


\section{Introduction}

\section{Introduction}

1.1 Motivation ............. 2

1.2 Definition of a glass . . . . . . . . 3

1.3 Strong and fragile liquids ....... . . . . . 4

1.4 The glass transition . . . . . . . . . . . . 5

1.5 The Kauzmann paradox . . . . . . . . 6

2 Models of glasses

2.1 Mode-coupling theory (MCT) . . . . 7

2.2 Random first-order transition theory (RFOT) ............... 8

2.3 The potential energy landscape (PEL) . 9

2.4 The free energy landscape . . . . . . . . 12

2.5 Discussion . . . . . . . . . . . 12

3 Theoretical background

13

3.1 Thermodynamic formalism . . . . . . 13

3.2 Density of configurational states and entropy .............. 14

3.3 Basin free energy . . . . . . . . . 15

\section{Sampling schemes}

4.1 Reverse Monte Carlo (RMC) . . . . . . 15

4.2 Molecular dynamics: aging versus equilibrium . . . . . . . . . . . 15

4.3 Density of states Monte Carlo . . . . . . 18

4.4 Continuous Random Networks (CRN) . 18

4.5 Stochastic Quenching (SQ) . . . . . 18

4.6 Activation-Relaxation Technique (ART) 19

4.7 Discussion . . . . . . . . . . . . 19

5 Theoeretical characterisation $\quad 19$

5.1 Structure and packing . . . . . . . . 20

5.2 Density of states . . . . . . . . . . . . . 21

5.3 Normal mode analysis . . . . . . . . . 21

5.4 Activated dynamics . . . . . . . . . . 22

5.5 Network topology . . . . . . . . . . . . . . 23

5.6 Discussion . . . . . . . . . . . . 24

6 Model Systems

6.1 The binary Lennard-Jones (BLJ) glass . 25

6.2 Amorphous silica . . . . . . . . . . . . 27

6.3 Hard sphere model . . . . . . . . . . . . 28

6.4 Discussion . . . . . . . . . . . . 28

7 Ab initio models

8 Glass-forming ability

9 Conclusions

30

\subsection{Motivation}

One of the biggest challenges in condensed matter physics is the modelling of amorphous solids, which lack the long range order characteristic of crystalline solids. When modelling bulk crystals, it is possible to exploit the translational symmetry of the system, allowing the construction of a unit cell subject to periodic boundary conditions, i.e. the translation of a basis along lattice points. This method does not trivially map to amorphous phases which contain no such symmetries, although a large enough unit cell will capture to some extent the short and medium range disorder in spite of the artificial periodic boundary conditions. This, however, comes at great computational expense. For example, first principles calculations in the framework of density functional theory scale as $N^{3}$ for a unit cell containing $N$ atoms, while the scaling of higher level quantum mechanical methods is even worse. For reasons elucidated below, interest in glasses is increasing, and with it the need for a complete theoretical model, which is not currently available.

One such reason is the new class of materials called bulk metallic glasses. Metal atoms have weak orientational constraints in the solid state, and therefore tend to crystallise easily. Certain alloy compositions, such as $\mathrm{Cu}_{64} \mathrm{Zr}_{36}$ have a much lower tendency to crystallise, i.e. a good glass-forming ability [1], while others, such as $\mathrm{Ni}_{64} \mathrm{Zr}_{36}$ tend to form ordered structures [2]. Bulk metallic glasses are currently of particular interest due to their unique physical properties, such as high elastic moduli or plasticity [3] and the potential for surface smoothness not limited to crystal facets. They lack an intrinsic length scale (grain size in the case of crystalline structures, or chain length in the case of polymer glasses), and can in principle be shaped at the atomic level [4]. Finding compositions with good glass forming ability is a timeintensive task, with one composition taking around a day to fabricate; this trial-and-error process could be greatly streamlined using modelling methods [4].

Another reason is the development of physical vapour deposition as a technique for synthesising glasses with extraordinary thermodynamic and kinetic stability [5-8]. Until recently, such ultrastable glasses were limited to complex molecular and polymer glasses; more recently however, vapour deposition has allowed the preparation of ultrastable metallic glasses [9]. When formed from the liquid phase, the properties of a glass depend strongly on its thermal history, i.e. its cooling rate, and it cannot be assumed to be at equilibrium, since glasses are known to "age", or find more thermodynamically favourable states as 
time passes. Recent developments in vapour deposition techniques have allowed the synthesis of ultrastable glasses occupying equilibrium states equivalent to a supercooled liquid aged for thousands of years or cooled at a rate 19 orders of magnitude slower than current methods allow [10]. Although aging is believed to allow a glass to find more stable states, experimental determinations of the enthalpies of ultrastable metallic glasses formed by vapour deposition suggests that their stability is not necessarily a result of being in the lowest thermodynamic state [9]. There are many empirical measures of glass forming ability (see section 8), but there is not coherent microscopic structural explanation that accounts for the differences between compositions. Furthermore, it is possible to synthesise stable glasses from compositions with low phenomenological glass forming ability such as $\mathrm{Fe}_{1-\mathrm{x}} \mathrm{C}_{\mathrm{x}}$ using thin film vapour deposition techniques[11]. This suggests that there is something missing in the various empirical measures of glass forming ability, and a more fundamental description is required.

An example of a good glass former is $\mathrm{B}_{2} \mathrm{O}_{3}$. A $\mathrm{B}_{2} \mathrm{O}_{3}$ melt does not crystallise even when seeded with a crystallite of the stable phase, as a result of the low driving force and slow kinetics; its crystallisation can only be induced at high pressure, when enthalpic considerations force the structure to adopt an optimal packing [12]. This leads to the question of which parameters define a good glass former. In general, we describe a material that has a high tendency to form a glass instead of a crystal when quenched. There are many empirical measures of glass-forming ability [13-15], but none are derived from an atomistic description of a glass or offer a physical insight into the structure of a glass. What is the driving force in the glass transition? Is it possible to characterise a good glass former by the potential energy landscape alone, or do free energy considerations need to be taken into account? There is strong evidence that composition and stoichiometry control glass forming ability rather than simple physical and topological considerations [16]. Is there a parameter that can be used to theoretically distinguish a good glass forming composition from a crystal forming composition? Which variable, if any, describes glass forming ability? In this review, we take a step towards answering these questions, and in doing so, demonstrate that the potential energy landscape is a useful paradigm for predicting the characteristics of amorphous systems.

The potential energy landscape (PEL) is a simple concept: for a system of $N$, atoms, the potential energy is defined for each atomic configuration, i.e. at each point of the $3 N$-dimensional configuration space. The resulting hypersurface contains minima and saddle points, at which the gradient of the potential energy (the forces) are zero. The minima correspond to stable and metastable atomic configurations, and the saddle points to transition states between minima. Common atomistic calculations examine local features of the landscape, such as a minimum (zero temperature structure optimisation) or a limited region determined by the temperature (molecular dynamics), but our aim is to link collective and statistical features of the PEL with the properties of amorphous materials and glasses.

This review is organised as follows. In section 1 we introduce the topic of computational modelling of glasses and the problems therein, setting some basic defintions. In section 2, we introduce the theoretical frameworks most commonly used to model glasses, with an emphasis on the potential energy landscape. In section 3 we discuss the construction of the potential energy landscape paradigm in a more rigorous way, focusing on the statistical mechanics required to link microscopic computer models to thermodynamic quantities. In section 4, we summarise the methods for sampling the potential energy landscape, i.e. how to generate atomistic models of glasses. In section 5 we describe the methods by which such structural models can be analysed. Simple empirical models of glasses are discussed in section 6, followed by an extension to the use of first principles electronic structure calculations for higher quality models in section 7. Finally, we discuss possible methods and parameters for distinguishing good crystal-formers from good glass-formers in section 8 and finish with some conclusions in section 9 .

\subsection{Definition of a glass}

A glass is an amorphous material for which, below a crossover temperature, the timescale of its dynamical properties suddenly and dramatically increase beyond the experimental timescale. Below the transition temperature, all molecular motion ceases, except for thermal vibrations [17] (this is not strictly true, a counterexample being long timescale Johari-Goldstein $\beta$-processes [18] — see section 3.1; however, this is not enough to induce a viscous flow). There are numerous possible definitions for the crossover temperature, discussed in section 1.4.

Generally, one can distinguish between two types of structurally (as opposed to magnetically) disordered system [19]. The first type is systems with configurational but not structural disorder, such as random alloys, in which atomic positions are topologically fixed by the underlying Bravais lattice, but the distribution of atoms on the lattice is random. Crystalline systems containing defects such as vacancies, interstitial atoms and dislocations also belong to this category, since removal of these defects will restore a disordered material to a crystalline 
state. Atomic vibrations and local atomic relaxations result in displacements from the ideal lattice positions, but they preserve the translational symmetry of the underlying Bravais lattice "on average," and the theory of such systems can be built using the usual formalism of reciprocal space [20]. The second type is comprised of structurally or topologically disordered systems, where atomic positions cannot be associated with any underlying Bravais lattice. A straight line through a perfectly amorphous material in an arbitrary direction will be divided by the atoms into "irregular intervals of random sequence" [21].

A snapshot of the instantaneous atomic configuration of any amorphous solid is indistinguishable from that of a liquid. The glass transition is often attributed to a dynamic heterogeneity [22], since there has been little evidence of any change in structure as indicated by the static structure factor $S(q)$ across the glass transition temperature (although this is not true for some systems [23]). However, a recent study suggests that the dynamic heterogeneity may have an origin in Isinglike criticality in static structural order [24]. A glass does not flow like a liquid, but its diffusive process are very different to those that occur in crystals [25]. Instead of single-atom hops, atoms in glasses undergo complicated collective motions of ten or more atoms [26]. The diffusive processes can be divided into three types of local excitations: tunneling systems, excess vibrations and local relaxations [25]. Within the soft potential model whichis parameterised using experimental data, these effects are included in collective motions of 20 or more atoms [27].

The difference between an amorphous solid and a glass is debatable since the terms are often used interchangeably in the literature. When a liquid is cooled below its experimental glass transition temperature, $T_{g}$, the timescale of its dynamical properties of a liquid suddenly and drastically increase. Its transport properties diverge from the Arrhenius law, for which the rate of a process is proportional to $e^{-E_{a} / k_{\mathrm{B}} T}$ where $E_{a}$ is some activation energy. Below $T_{g}$, the viscosity of the system is above approximately $10^{13} \mathrm{P}$, and the system can no longer reach thermal equilibrium since the relaxation timescale exceeds the experimental timescale (of the order 100s) [28]. A glass can therefore be considered to be a special case of amorphous solid that undergoes the glass transition when the liquid phase is cooled.

According to Ediger and Harrowell [29], there are three characteristics of glasses formed from supercooled liquids (liquids cooled below their melting temperature without undergoing crystallisation): firstly a huge increase in shear viscosity, secondly a temperature dependence of the entropy manifested in the socalled Kauzmann paradox [30], and thirdly dynamic heterogeneity, suggesting some kind of solid-liquid coexistence, a more recent addition to menagerie of glass theories [31].

Another definition is the transition from an ergodic state to a non-ergodic state, i.e. from a liquid state which will in principle explore the entire configurational space given enough time, to a glassy state which is trapped in a single minimum with insufficient kinetic energy to surmount any activation barriers. A more phenomenological distinction is the appearance of a non-zero shear modulus on transition to a glassy state.

\subsection{Strong and fragile liquids}

Glasses-forming liquids can broadly be categorised on a scale, for which the extrema are strong liquids and fragile liquids. Strong liquids tend to have open network structures with strong directional bonds which resist temperature-induced structural change. Fragile liquids, on the other hand, are characterised by less directional interactions (for example, Ionic and Van der Waals) [32]. This delineation is not arbitrary; strong liquids such as $\mathrm{SiO}_{2}$ have small or undetectable changes in their heat capacity $C_{p}$ in the temperature range over which the relaxation timescale crosses the experimental threshold. In such cases, distortion of the structure is restricted by strong directional bonds, constraining the $\mathrm{Si}$ atoms in $\mathrm{SiO}_{2}$ to be tetrahedrally coordinated, and resulting in a low density of configurational states. For a fragile liquid, the degeneracy associated with an increase in energy is much higher, and the structure is "smeared" out over a continuous temperature range. $C_{p}$ must be proportional to $k \ln W$, where $W$ is the density of states, hence the sharp increase in heat capacity at the glass transition [33]. Essentially, a fragile liquid samples more metastable structures.

Qualitatively, the two can be distinguished by means of an Angell plot (figure 1) of the logarithm of the viscosity against scaled temperature. The most widely used metric of fragility is the steepness index $m$, or the slope of the Angell plot [34]:

$$
m=\left.\frac{d \log \langle\eta\rangle}{d\left(T_{g} / T\right)}\right|_{T=T_{g}} .
$$

It has recently been shown that the fragility of a liquid can be predicted using the linear elasticity of the glass, 'a purely local property of the free energy landscape' [35]. Quantitatively, the distinction between strong and fragile liquids arises in the temperature dependence of the single particle diffusivity $D$ (which can be evaluated from molecular dynamics simulations) or equivalently the viscosity $\eta$. For strong glasses such as $\mathrm{SiO} 2, \eta$ has an Arrhenius form, suggesting well-defined energy barriers 


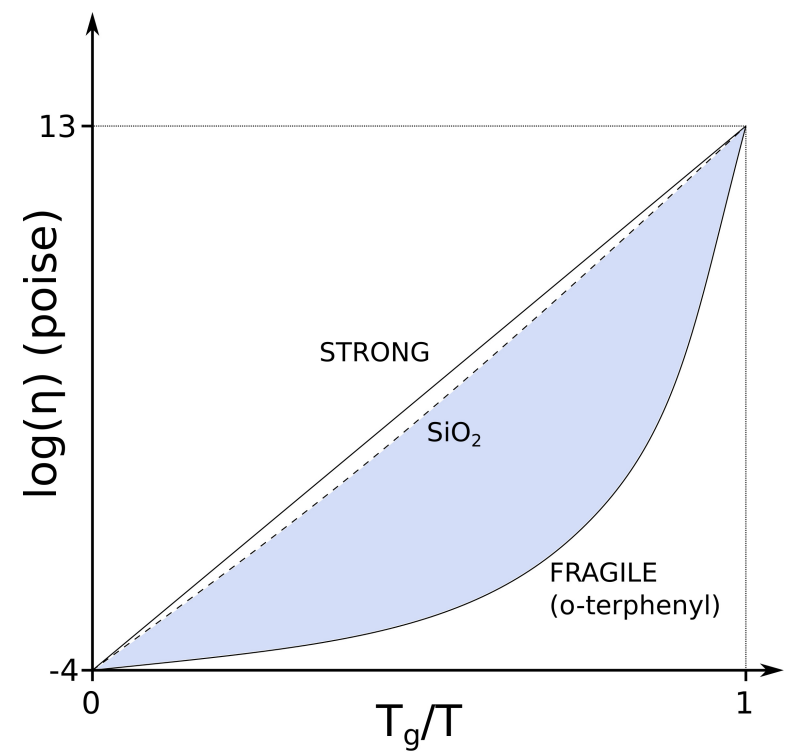

Figure 1. The Angell plot of viscosity $(\eta)$ against scaled temperature (adapted from reference [12]). Strong liquids tend to be close to a straight line, while fragile liquids are represented by lines that curve towards the bottom-right. Most liquids exist in the shaded region, between the strongest (silica) and the most fragile (o-terphenyl).

to diffusion, while weak liquids have a $\eta$ that increases at a greater, non-Arrhenius rate. This has been attributed to a collective rearrangement of groups of particles in a fragile liquid, compared with small local rearrangements due to the strong directional bonding in a strong liquid [36]. This fragile-strong behaviour is thought to be intimately connected with the phenomenon of polyamorphism in glasses [37]. A dynamical crossover temperature $T_{B}$, well above $T_{g}$, was established as the strong-fragile transition for oterphenyl recently [38], and it has also been suggested that a liquid-liquid phase transition may result in the change in fragility [39].

How does fragility affect glass-forming ability? The two should not be conflated, since their relationship is not simple, and the advent of vapour deposition methods for preparing ultra-stable glasses has made it even more unclear. Fragile liquids are better glass formers when cooled from the liquid phase, and all molecular systems which have been shown to form stable glasses have intermediate-to-high fragilities $(m)$, although it has been shown that organic molecules with low fragilities can form stable glasses via vapour deposition [8].

\subsection{The glass transition}

According to Angell, the essential feature of the glass transition is the appearance of a huge gap between the vibrational and structural equilibration timescales, of up to 17 orders of magnitude [12], but it is difficult to associate this change with a thermodynamic phase transition. The glass transition resembles a second order phase transition in the Ehrenfest sense, with continuous volume and entropy changes, but it cannot be defined as a genuine phase transition since crossover temperature is cooling rate-dependent [17]. It is not generally thought to be a thermodynamic first order transition, since no latent heat has been measured. In the case of metallic glasses, this question is still open, since the the latent heat may be small and therefore difficult to measure, and there is evidence for a first order transition from experiment [40] and simulation [41]. Some simulations suggest that it may be a different type of transition, a non-equilibrium first order transition in trajectory space, controlled by variables that drive the system out of equilibrium [42].

The transition from a supercooled liquid to a glass is generally vaguely defined, and it can be characterised by a number of different temperatures or temperature regimes. There are three transition temperatures that commonly appear in the literature, the experimental glass transition temperature $T_{g}$, the Kauzmann temperature $T_{K}$ (see section 1.5) and the mode-coupling transition temperature $T_{\mathrm{MCT}}$ (see section 2.1).

The experimental glass transition temperature $T_{g}$ is defined as the temperature at which the liquid, on cooling, exhibits a sharp decrease in heat capacity $\left(C_{p}\right)$, to a value approaching that of the crystalline state. There is no standardised value for the cooling rate, but $T_{g}$ is usually defined as the temperature at which the onset of the $C_{p}$ increase occurs during the heating of a glass at a rate of $10 \mathrm{~K} \mathrm{~min}^{-1}$. Despite an apparent lack of consistency, $T_{g}$ is easy to determine, and there is often good agreement, to within $2 \mathrm{~K}$ between independent groups, when the only reproduced condition is the cooling rate [43]. This temperature is, however, insufficient to describe all aspects of the glass transition. The aforementioned timescale difference can vary by as much as 10 orders of magnitude between $T_{g}$ and $1.1 T_{g}$ [12]. The heat capacity peak, and plateau in thermal conductivity are generally assumed to result from a universally observed anomaly in the vibrational density of states of glasses, identified by an excess of low energy states above the acoustic modes, called the "boson peak." The boson peak has been linked to a transverse acoustic van Hove singularity in the corresponding crystalline phase [44].

In the vicinity of $T_{g}$, the relaxation behaviour of a supercooled liquid diverges from the exponential Arrhenius form, instead conforming to a stretched exponential of the form $\exp \left[-(t / \tau)^{\alpha}\right]$ (often described using the empirical Vogel-Fulcher-Tamman, or VFT law; see section 1.5), where the stretching exponent 
$\alpha$ lies between 0 and 1 [45]. It has been suggested that a discontinuity in the relaxation time at the crossover from the Arrhenius to the VFT regime (as the temperature decreases below $T_{g}$ ) might constitute the aforementioned phase transition. Measurements on three good glass formers reveal a sharp crossover between the two regimes with a width of less that $15 \mathrm{~K}$, that cannot be accounted for by phenomenological models [46].

The definition of a laboratory glass transition remains somewhat vague, since the properties of the glass formed vary with the cooling rate, with a faster cooling resulting in a higher $T_{g}$. An ideal glass transition can be defined in the limit of an infinite cooling rate; above the ideal transition temperature, the behaviour of the system is ergodic, and it has time to explore every possible metastable structure, until it ends up in the most thermodynamically favourable non-crystalline state at the global minimum state of the potential energy landscape [47]. The character of this non-crystalline minimum, or ideal-glass state remains unclear; for example, is there some local degree of crystallinity, and if so, to what extent are crystalline inclusions allowed in an amorphous state [48]?

\subsection{The Kauzmann paradox}

Kauzmann defined an ideal glass transition temperature, resulting from a paradox [30]. Using thermodynamic data from a variety of molecular glass-formers, he demonstrated that the excess entropy of the liquid $\left(S_{e x}\right)$ with respect to the entropy of the crystal $\left(S_{\text {cryst }}\right)$ vanishes at some temperature below $T_{g}$, named $T_{K}$. In other words, a graph of $S_{\text {ex }} / S_{\text {cryst }}$ against temperature reaches zero at a finite temperature [12] $T_{K}$, at which point the liquid and crystal have equal entropy, as illustrated in figure 2 .

Decomposing the total entropy into vibrational $\left(S_{\mathrm{vib}}\right)$ and configurational $\left(S_{\text {conf }}\right)$ components, we have

$$
S=S_{\mathrm{vib}}+S_{\mathrm{conf}}
$$

and we define the excess entropy as,

$$
S_{\text {ex }}=S_{\text {liq }}-S_{\text {cryst }}
$$

It is commonly assumed that the vibrational contributions are similar for liquid and crystalline phases, such that,

$$
S_{\mathrm{ex}} \simeq S_{\mathrm{liq}, \mathrm{conf}}-S_{\mathrm{cryst}, \mathrm{conf}}=\Delta S_{\mathrm{conf}}
$$

It seems counterintuitive that a liquid or amorphous solid can have a lower configurational entropy than a crystal below $T_{K}$, and this is the apparent paradox. However, there is no general principle constraining the entropy of a liquid to be greater than that of a
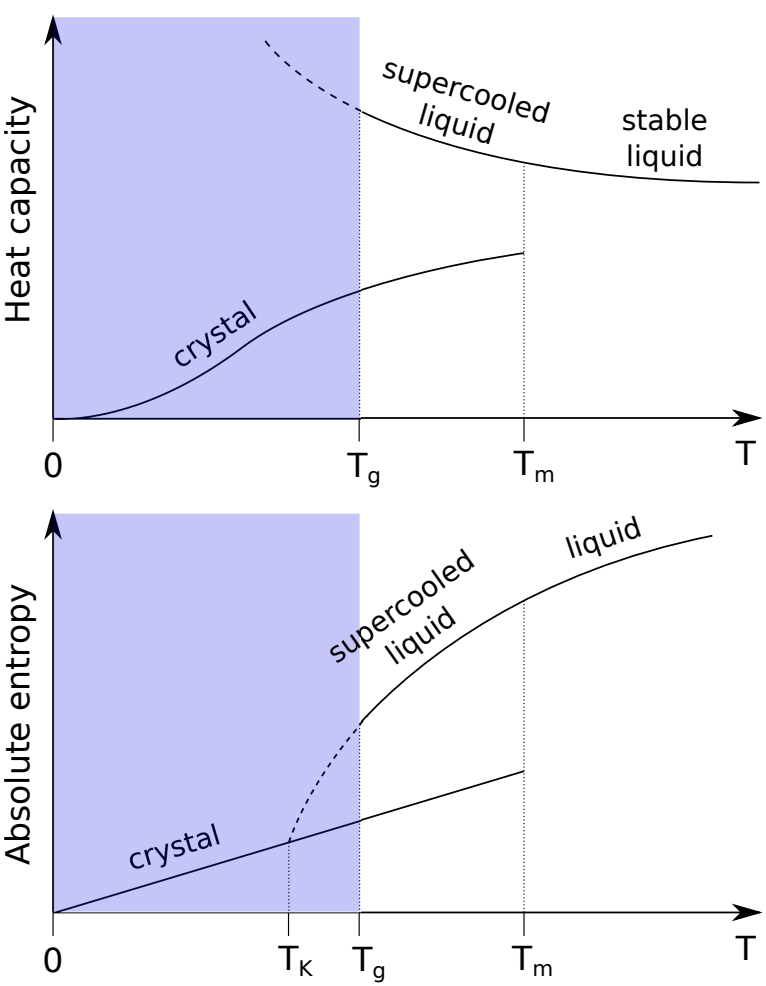

Figure 2. Illustration of the Kauzmann paradox (adapted from reference [50]), showing the increase in heat capacity as a liquid is cooled below its melting temperature $T_{m}$, and the crossing of the absolute entropy curves for the liquid and the crystal at the Kauzmann temperature $T_{K}$. The shaded region is experimentally inaccessible for a glass due to the slowing of dynamics below $T_{g}$.

crystal [49]; indeed, Kauzmann notes that it is possible to conceive a situation at lower temperatures, where tighter binding of a molecules in a highly strained liquid structure would result in high vibrational frequencies and a lower density of vibrational states.

It turns out that $T_{K}$ correlates impressively well with the divergence temperature $T_{0}$ of the phenomenological Vogel-Fulcher-Tammann (VFT) law. It introduces a different measure of fragility in the form of the parameter $D$, which quantifies the deviation from the Arrhenius law [51],

$$
\eta=\eta_{0} \exp \left(\frac{D T_{0}}{T-T_{0}}\right)
$$

where $\eta$ is the viscosity. The correlation is reflected in the excellent agreement between $T_{g} / T_{K}$ and $T_{g} / T_{0}$ [43], and the fact that $T_{K} \simeq T_{0}$ for a wide range of molecular and covalent glass formers [52]. However, this relationship is not universal, and breaks down for many glass formers. Here, there is instead a positive correlation between $T_{K} / T_{0}$ and $D$ [53]. This relationship is attributed to short range ordering in the case of strong liquids, modelled by the formation of the glass from superatomic rather than atomic units. 
When modelling glasses, it should be noted that neither the vibrational nor configurational entropy is proportional to the excess entropy (as demonstrated for liquid Se), and that the anharmonic contribution to the vibrational entropy cannot be ignored $[54,55]$.

In practice, $T_{K}$ is an experimentally inaccessible quantity because the glass transition intervenes before the excess entropy can reach zero, but its value can be inferred from the VFT equation. For the same reason, the Kauzmann temperature has not been directly observed in dynamical simulations, and its existence has only been inferred from the extrapolation of high temperature data [56].

The existence of the Kauzmann entropy crisis is supported by the Adam-Gibbs equation which relates the relaxation timescale to the configurational contribution to the excess configurational entropy $S_{\text {conf }}$ [36]:

$$
\tau \propto \exp \left(\frac{C}{T S_{\text {conf }}(T)}\right)
$$

This expression is based on the concept of weakly interacting subsystems within the glass, expressing the cooperative motion of distinct spatial regions in addition to individual atoms. Although it has been argued that the underlying arguments are weak, it demonstrates a divergence in relaxation time as the excess configurational entropy tends to zero, and has also been shown to be in good agreement with experiment.

The Kauzmann paradox can be viewed as evidence that the glass transition is essentially a thermodynamic phenomenon masked by slow kinetics [12], and implies that it may be possible to predict glass forming ability via the potential energy landscape rather than the free energy landscape.

\section{Models of the glasses and the glass transition}

In this section, we discuss the most important theoretical frameworks used to describe glasses and the glass transition. We mention mode-coupling theory and random first-order transition theory only briefly though, since they are beyond the scope of this review, and are discussed in detail elsewhere [57-60].

\subsection{Mode-coupling theory (MCT)}

The mode-coupling theory, developed independently by Leutheusser [61] and Bengtzelius et al. [62] characterises the dynamics of the glass transition using the kinetics of a simple hard sphere model. [59],

Starting from the density of particles $\rho$ in a liquid

$$
\rho(\boldsymbol{r}, t)=\sum_{i} \delta\left(\boldsymbol{r}-\boldsymbol{r}_{i}(t)\right)
$$

which has the Fourier transform,

$$
\rho_{\boldsymbol{k}}(t)=\sum_{i} \int d \boldsymbol{r} e^{i \boldsymbol{k} \cdot \boldsymbol{r}} \delta\left(\boldsymbol{r}-\boldsymbol{r}_{i}(t)\right)=\sum_{i} e^{i \boldsymbol{k} \cdot \boldsymbol{r}} .
$$

The correlation function of interest is the dynamic structure factor $F(\boldsymbol{k}, t)$ :

$F(\boldsymbol{k}, t)=\frac{1}{N}\left\langle\rho_{-\boldsymbol{k}}(0) \rho_{\boldsymbol{k}}(t)\right\rangle=\frac{1}{N} \sum_{i j}\left\langle e^{-i \boldsymbol{k} \cdot \boldsymbol{r}_{i}(0)} e^{i \boldsymbol{k} \cdot \boldsymbol{r}_{j}(t)}\right\rangle$.

The MCT equation (derived in detail in reference [59]) is the non-linear equation of motion of this time and wave vector dependent correlation function.

$$
\frac{d^{2} F(q, t)}{d t^{2}}+\frac{q^{2} k_{\mathrm{B}} T}{m S(q)} F(q, t)+\int_{0}^{t} d \tau K(q, t-\tau) \frac{\partial F(q, t)}{\partial \tau}=0 .
$$

Recasting this using $\Phi(t) \sim F(k, t)$ neglecting the coupling wave vectors results in the expression,

$$
\frac{\partial^{2} \Phi(t)}{\partial t^{2}}+\Omega_{0}^{2} \Phi(t)+\lambda \int_{0}^{t} d \tau \Phi^{2}(t-\tau) \frac{\partial \Phi(\tau)}{\partial \tau}=0 .
$$

At $t=0, F(\boldsymbol{k}, t)$ is identical to the static structure factor $S(k)$ of a liquid, which can be measured experimentally. At high temperatures, above the melting point, $\Phi(t)$ decays exponentially with time, but for supercooled liquids, it undergoes a multistep relaxation (figure 3). At short times, free and collisional events result in the rapid decay in region I, referred to as $\alpha$-relaxation. The plateau of region II is a result of the trapping of particles in cages formed by their nearest neighbours, and the subsequent decaying regime corresponds to cage-breaking events, is known as the $\beta$-relaxation regime. At long times, the $\beta$-relaxation takes a stretched exponential form.The solution for the case of a glass is sketched in figure 3 . MCT is characterised by a sharp transition from an ergodic regime to a non-ergodic regime as the temperature is decreased below the mode-coupling temperature $T_{\mathrm{MCT}}$. This behaviour is not system dependent, and applies to both metallic and nonmetallic glasses [25].

MCT makes some very detailed predictions that have been experimentally verified (particularly with respect to $\alpha$ and $\beta$-relaxation), and is generally valid in the vicinity of the glass transition. The MCT singularity is not equivalent to the laboratory glass transition described in section 1 , since it occurs $30-50 \mathrm{~K}$ higher than $T_{g}$, and it appears to work considerably better for fragile liquids than for strong liquids [28]. Moreover, its most obvious failing is the prediction of a sharp transition, contrary to what is observed in experiments. It has been successfully tested against molecular dynamics simulations for a variety of systems, including binary Lennard-Jones soft spheres 

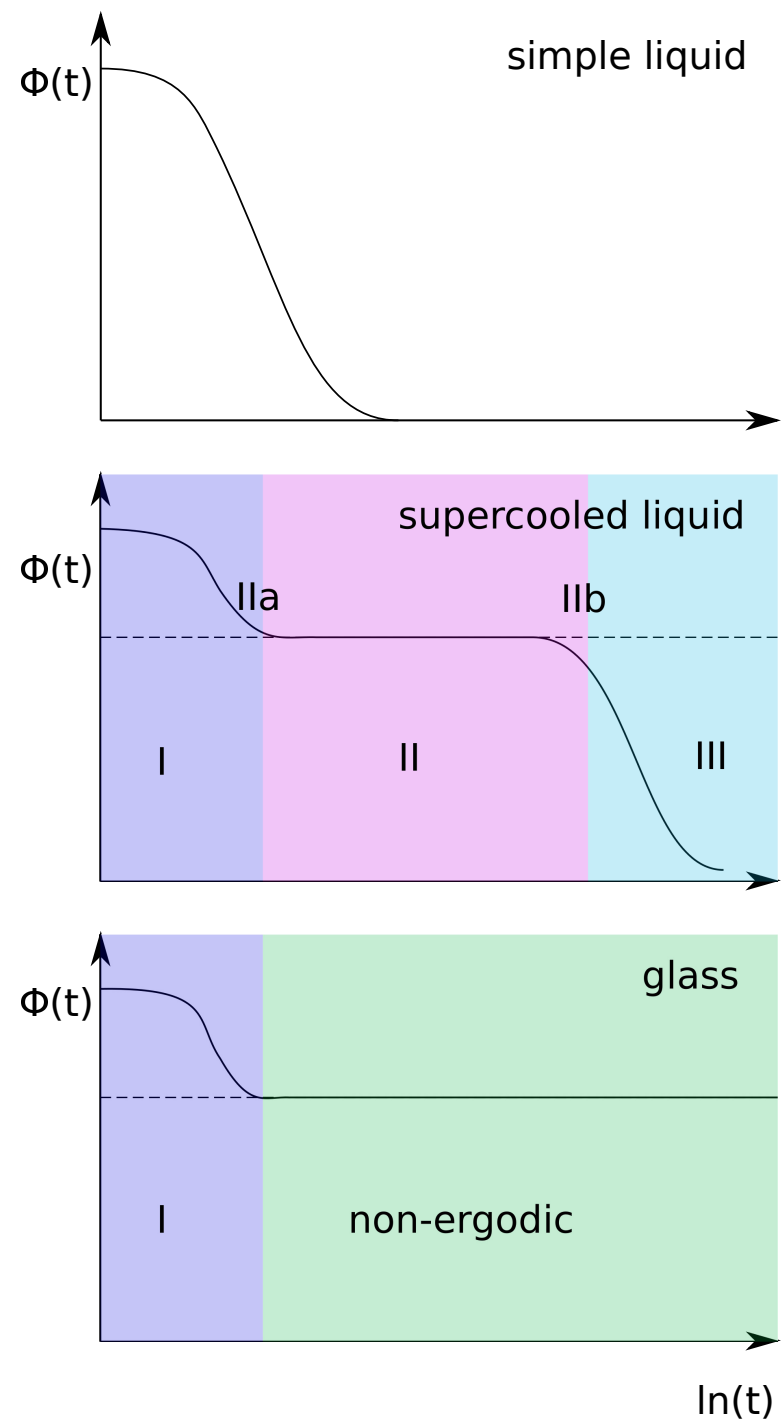

Figure 3. Wave vector-independent solutions to MCT equation (11) for three cases (adapted from reference [59]). A simple liquid is characterised by exponential decay in the correlation function. A supercooled liquid has three distinct regimes: I. short timescale decay from free and collisional events; II. intermediate plateau, particles are trapped in cage comprised of nearest neighbours ( $\beta$-relaxation); III. long timescale nonArrhenius (stretched exponential) decay. The decay in the transitions to and from regime II (IIa and IIb) obey power laws with respect to time. The glass has a singularity in the solution of the MCT equation at $T_{\mathrm{MCT}}$; this results in the "freezing" of the structure in the non-ergodic regime.

$[63,64]$, binary hard spheres [65] and liquid silica [66]. In the latter, MCT calculations are performed using only structure factor data from molecular dynamics, and while there are discrepancies (in particular for the crossover temperature), a remarkable degree of quantitative agreement is shown for this ab initio theory. A more complete summary of the current state of MCT is available in references [58, 59].

\subsection{Random first-order transition theory (RFOT)}

RFOT is characterised by two distinct transition temperatures: a dynamical transition corresponding to ergodicity-breaking which can be formally identified with the mode-coupling temperature, and a second transition at a lower temperature corresponding to a thermodynamic phase transition to a glassy phase characterised by a discontinuity in an order parameter, but no latent heat [67]. When a liquid is cooled below its glass-transition temperature, it can be considered to undergo a phase transition analogous to the first order crystallisation transition. Particle motion is a superposition of translations to a new position and vibrations about a mean position; above the transition, the motion is dominated by translations, and below it is dominated by vibrations, with only occasional moves. Below the glass transition temperature, the particle is essentially trapped in a cage formed by its nearest neighbours and is unable to move due to the high vacancy formation energy. Crystals have a periodic density profile $\rho(\boldsymbol{r})$, but this can be generalised to the following for non-periodic structures [68].

$$
\rho(\boldsymbol{r})=\rho\left(\boldsymbol{r},\left\{\boldsymbol{r}_{i}\right\}\right)=\sum_{i}\left(\frac{\alpha}{\pi}\right)^{3 / 2} e^{-\alpha\left(\boldsymbol{r}-\boldsymbol{r}_{i}\right)^{2}}
$$

Here, $\left\{\boldsymbol{r}_{i}\right\}$ is the set of coordinates of a generic aperiodic lattice of average lattice spacing $a$ and density $n=1 / a^{3} . \quad \alpha$ is a variational parameter describing the localisation of a particle in a cage formed of its neighbours, for which a completely delocalised liquid has $\alpha=0$, and an (meta)stable structure has $\alpha=\alpha_{0}$. The quantity $1 / \sqrt{\alpha_{0}}$ is the vibrational amplitude of the metastable structure and is directly comparable to the Lindemann length $d_{L}$,

$$
d_{L}=\frac{1}{\sqrt{\alpha_{0}}}
$$

The Lindemann ratio $d_{L} / a$ is approximately 0.1 for crystals, and roughly defines the point at which the vibrational motion becomes comparable to the nearestneighbour distance, resulting in melting. It turns out to be universal, and only weakly dependent on the nature of the bonding [69]. A liquid-crystal transition is first order, and similarly a liquid-glass transition is first order in $\alpha$; however, in contrast with a single distinct crystal structure, a glass can be considered to be an ensemble of metastable structures with a random distribution of free energies (hence the name RFOT). This results in a multiplicity of distinct structural states with an infinite lifetime, thus there is no latent heat, but instead a heat capacity discontinuity.

RFOT is built on the density-functional theory of freezing (a static treatment of mean-field theory), 
and turns out to be formally equivalent to modecoupling theory (a perturbative treatment of meanfield theory; see section 2.1) [70]. In the RFOT, glassy states correspond to the minima of the free energy $F$ as a functional of inhomogeneous number density $n(\boldsymbol{x})$, corresponding to a non-uniform density field. Starting from a random close packed structure, a trial density field, containing a parameter that describes both uniform and non-uniform states, is constructed. It is possible to calculate the free energy as a function of this parameter, such that it is possible to find the point at which an non-periodic solution becomes possible. The free energy functional can be approximated to [71],

$$
\begin{aligned}
F[n(\boldsymbol{x})] & =F_{0}[n]+F_{\mathrm{int}}[n] \\
& =\int d \boldsymbol{x} n(\boldsymbol{x})[\ln n(\boldsymbol{x})-1] \\
& -\frac{1}{2} \int d \boldsymbol{x} \int d \boldsymbol{x}^{\prime} n(\boldsymbol{x}) C\left(\boldsymbol{x}-\boldsymbol{x}^{\prime}\right) n\left(\boldsymbol{x}^{\prime}\right)+F_{\mathrm{uni}},
\end{aligned}
$$

in which $F_{0}$ is the ideal gas free energy functional, $F_{\text {int }}$ is the lowest order term in the expansion of interaction part of the free energy and $F_{\text {uni }}$ is a constant term contributing to the free energy of the liquid. $\quad C(\boldsymbol{x})$ is the liquid-state direct correlation function, a renormalised form of the bare interaction potential [69]; its Fourier transform $\tilde{C}(q)$ is related to the measurable static structure factor $S(q)$ (the Fourier transform of the radial distribution function) by

$$
n \tilde{C}(q)=1-\frac{1}{S(q)}
$$

A dynamical RFOT can also be derived, and the resulting dynamical equation is,

$\frac{\delta F_{s}}{\delta n_{s}(\boldsymbol{x}, t)}=k_{\mathrm{B}} T\left[\ln n_{s}(\boldsymbol{x}, t)-\int d \boldsymbol{x}_{1} C\left(\boldsymbol{x}-\boldsymbol{x}_{1}\right) n\left(\boldsymbol{x}_{1}, t\right)+\ldots\right.$

Glassy states are non-periodic solutions of

$$
\frac{\delta F[n]}{\delta n(\boldsymbol{x})}=0,
$$

An alternative description of the glassy state arises naturally from RFOT, the "mosaic scenario." Modecoupling theory is based on the concept of dynamic heterogeneity, and the glassy state is described by dynamical correlation functions; however, the mosaic scenario defines a static correlation length in an ensemble of inequivalent states below the modecoupling temperature [72]. Bouchaud and Biroli [67] construct a model starting with a non-periodic system consisting of a spherical region of Lennard-Jones particles of radius $R$ in a metastable configuration $\alpha$, with hard boundary conditions, i.e. the particles surrounding the sphere are frozen. The free energy cost of rearranging this configuration to another state, $\beta$, then consists of two opposing contributions: a surface tension component due to the change at the boundary, and an entropic contribution from the change in the configuration. The glassy state can be described by the length scale $\xi_{\mathrm{mos}}$ : if $R<\xi_{\mathrm{mos}}$, then the configuration may "flip" from $\alpha$ to $\beta$, but the pinning field of the frozen boundary will force it to flip back. For $R>\xi_{\text {mos }}$, a change in state is thermodynamically favoured, but takes a longer time due to the higher degeneracy, so $\xi_{\text {mos }}$ becomes the size of the cooperative region [72]. Therefore, large clusters are thermodynamically in a liquid state, while a glass is formed of a "mosaic" of mesoscopic, locally metastable domains, separated by strained but mobile walls. The relaxation within a mosaic domain is an activated process driven by the configurational entropy [51, 67]. These domains have been shown in simulations to change from sphere-like at low temperatures to string-like or "fractal" at $T_{\mathrm{MCT}}$ [73].

There is some controversy over the validity of the mosaic scenario, with some studies suggesting a possibly contradictory one-state scenario [72, 74], and others producing evidence in its favour [75]. Interestingly, these domains have been directly observed [76] and their size is consistent with predictions, around 90200 molecules [69, 73]. Bhattacharyya et al. have attempted to unify RFOT and MCT to describe the dynamics at low temperatures as well as around $T_{\mathrm{MCT}}$, and have thus been able to predict the fragility parameter $D$ and the stretching exponent in the non-Arrhenius regime [77].

\subsection{The potential energy landscape (PEL)}

The static structure, or state of a system of $N$ particles ] can be described as a point in a $3 N$-dimensional configuration space; its dynamics can then be viewed as as the motion of a state point $\boldsymbol{r}$ at an elevation of potential energy $\phi(\boldsymbol{r})$ above a $3 N$-dimensional energy landscape at zero temperature. This landscape is characterised by local minima for which $d \phi / d \boldsymbol{r}=$ 0 ; these generally represent metastable states of the system, and there exists a global minimum, which is the thermodynamically stable state of the system at absolute zero. The local minima, or basins, may be separated by barriers which represent the energy cost of breaking and making bonds to move from one (meta)stable state to another. A one-dimensional illustration is given in figure 4 .

If we are not interested in the kinetics of the system, it is helpful to consider only the basins of the system. Stillinger et al. argued first empirically and later more rigorously that the number of minima $\Omega(N)$ in an monatomic $N$-atom system follows the 


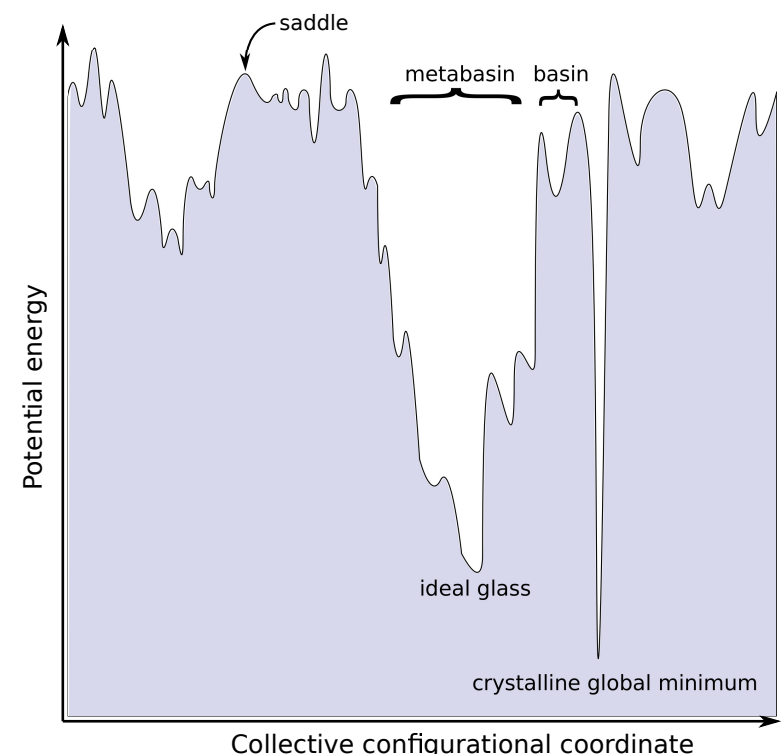

Figure 4. Schematic representation of the potential energy landscape (adapted from reference [78]). The horizontal axis represents the configurational degree of freedom, i.e. $3 N$ spatial coordinates. This landscape is more reminiscent of a glass former, since a randomly chosen coordinate on the $\mathrm{x}$-axis is more likely to correspond to a metastable amorphous state.

relationship [79, 80],

$$
\Omega(N) \propto N ! e^{\alpha N},
$$

where $\alpha$ is a positive constant in the large- $N$ limit. In nature, configurations are found in their lowest energy states because these have the largest Boltzmann weights in the canonical ensemble, which mitigates the timescale required for ergodicity. It is obvious that even for small finite systems, exhaustively sampling the basins in configuration space via computer simulation is impossible, necessitating a limited sampling. How to achieve this is a question that has yet to be answered fully, although some possible routes are suggested in section 2. Some small systems have been studied to the extent that their PEL is essentially well characterised, primarily in terms of the local minima. One such example is the thirteen-atom Morse potential $\left(\mathrm{M}_{13}\right)$ cluster [81]. Such studies have elucidated general features of the PEL, for example the existence of "funnels" in systems with a tendency to crystallise: these are collections of mutually accessible deep basins, possibly around the global minimum, and act as traps as the temperature is lowered.

How can one visualise such a high dimensional space? Qualitative schematic diagrams of one and two-dimensional surface are common as illustrations, but do not represent real PELs. Becker and Karplus introduced the use of disconnectivity graphs to map the partitioning of the PEL into local minima grouped into superstructures [82] (see figure 6). Each

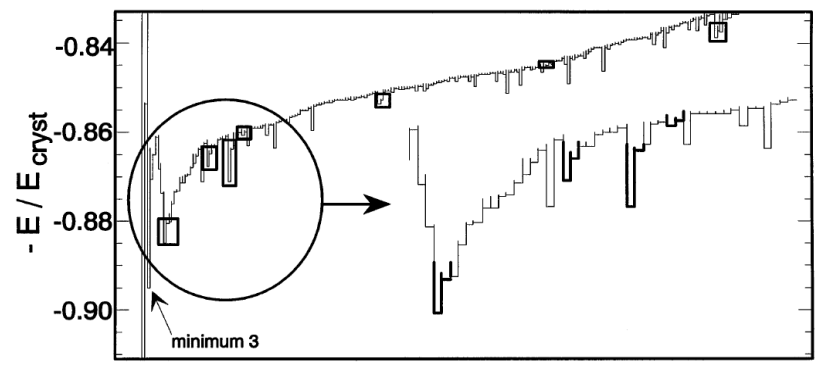

Figure 5. Heuer's scheme for projecting the PEL for a model system, a 32 atom binary Lennard-Jones glass (reprinted with permission from Phys. Rev. Lett. 78, 4051 (C)1997 American Physical Society [83]). Minima are grouped together along the horizontal axis based on their maximum potential energy, and barriers are indicated between minima. Note that adjacent minima on the diagram may be separated by large distances in configuration space.

"leaf" represents a distinct local minimum, while the branching structure describes how these local minima coalesce to form basins of basins (metabasins), and how these merge to form higher levels of structure. The effect of moving vertically up the disconnectivity graph is equivalent to increasing the temperature. As the kinetic energy in the system becomes large relative to the potential energy differences between the basins, the system does not "see" low energy basins with small barriers between them, but only the larger metabasin. If the temperature is raised enough, the system is free to explore the whole configuration space, and only sees one superbasin.

Angell explicitly proposes a connection between the topology of the PEL (namely the density of configurational states) and the fragility of the associated liquid [33, 43]. The PEL topology presents the resolution of the Kauzmann paradox: fragile liquids have a PEL with a statistically insignificant number (of order $N$ ) of deep minima, which contribute minimally to the density of structural states, i.e. the probability of finding a configuration of a specific energy on the PEL. This low degeneracy results in a low configurational entropy, comparable to that of a crystalline solid. Crystal structure prediction methods offer an insight into the character of the PEL for crystal formers: it is expected to consist of a super-basin, or "funnel," containing the global minimum crystalline state, which itself occupies a disproportionately large hypervolume of configuration space [84]. The strong liquid is somewhere in between, except the "ideal glass" state is expected to occupy a region with a low density of states (i.e. occupying a large volume of phase space). In the case of both glass-forming liquids, the crystalline minima occupy statistically insignificant portions of configuration space.

A comparison between the PELs of two cluster 
systems at the extrema of the glass forming scale gives us some clues about the differences between crystalforming systems and glass-forming systems. $(\mathrm{KCl})_{32}$ tends to form rock salt-like crystals, and the $\mathrm{Ar}_{19}$ system, which is rare gas-like, tends to form amorphous phases in spite of the existence of a double-icosahedral structure which is proposed to be the global minimum [85]. The $(\mathrm{KCl})_{32}$ PEL can be described as "step-like," whereas the $\mathrm{Ar}_{19}$ topology is "sawtooth-like." $(\mathrm{KCl})_{32}$ has a global minimum (rock salt) with basin energies increasing with distance from the global minimum, resulting in a funnel configuration. It crystallised even under rapid cooling of $10^{12} \mathrm{~K} \mathrm{~s}^{-1}$. In contrast, the energies of the minima of the $\operatorname{Ar}_{19}$ system differed only slightly apart from the global minimum; this system failed to find the global minimum even after slow cooling at a rate of $10^{9} \mathrm{~K} \mathrm{~s}^{-1}$.

The sawtooth/step dichotomy is a slightly simplistic scheme, but De et al. propose the distance-energy (DE) plot as a tool for distinguishing glassy clusters from non-glassy clusters on a similar basis [86]; the Euclidean root-mean-square distance (RMSD) in configuration space can be used as a "fingerprint" to determine the similarity of two phases, and as a parameter to define a 1D PEL [87]. In particular, the density of states in configuration space near the global minimum is much higher for glassy systems: fragility seems to be associated with a higher density of inherent states, lower activation barriers and higher vibrational frequencies [88]. A mathematical treatment of the density of states demonstrates that there is a strong correlation between ground state degeneracy and the roughness of the PEL [89], which fits well with the general picture that good glass-formers have a rough, highly degenerate PEL with no easily accessible ground state.

Doye and Wales [90] draw similarities between the potential energy surfaces and the free energy surface model used by Wolynes et al. to describe protein folding [91, 92]. The protein folding studies define temperatures $T_{f}$ at which the "native structure" (global minimum) becomes thermodynamically stable, and $T_{g}$ at which the protein is in a glassy state. A large ratio $T_{f} / T_{g}$ then ensures that the system becomes trapped in the native state rather than a local minimum. They demonstrate that increasing the barrier height in their simulations of Lennard-Jones clusters corresponds to increasing $T_{g}$ without changing $T_{f}$, thus reducing the rate of relaxation to the global minimum. Using this notation, $\mathrm{Ar}_{19}$ has a low ratio and $(\mathrm{KCl})_{32}$ has a high ratio.

Abraham and Harrowell make the interesting observation that inherent structures on the PEL of amorphous solids have non-zero shear stresses [93]. Considering the normal modes, they find that the only wave vector with a non-zero shear stress is $k=0$,

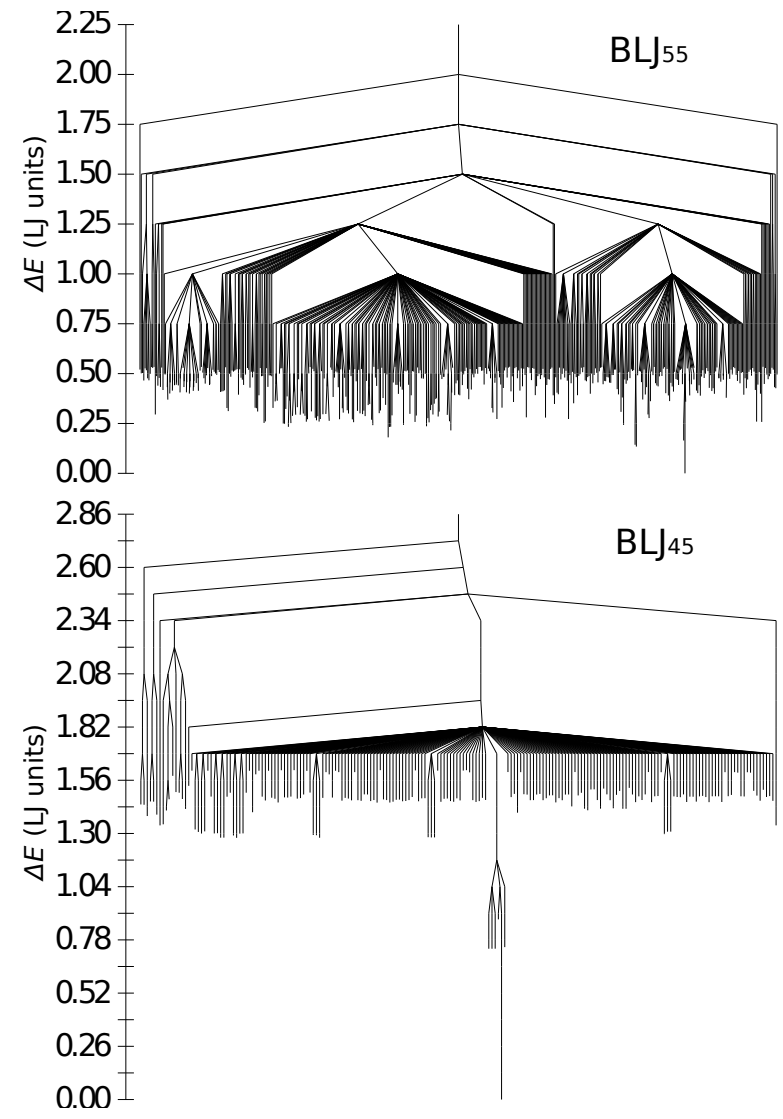

Figure 6. Disconnectivity graphs for the glassy binary LennardJones 55-atom cluster and the crystalline 45-atom cluster (reprinted with permission from Phys. Rev. Lett. 112, 083401 (C)2014 American Physical Society [86]). The glassy cluster has a multitude of near-degenerate states organised in many metabasins, while the crystalline cluster has one large metabasin and a distinct global minimum which is much lower in energy than the next lowest state.

i.e. fluctuations that span the simulation cell; this would be eliminated if the shape of the cell was allowed to change. Thus we arrive at the seemingly trivial conclusion that the inherent shear stress results from boundary conditions, and on a macroscopic level, the residual stress will exist in any extended aperiodic solid. One important conclusion to draw from this is that the inherent structures all correspond to rigid solid states since these must be able to support a stress by definition.

Doye et al. use disconnectivity graphs to map the PELs of various Lennard-Jones clusters, showing that certain cluster sizes have significantly different PELs (notably 31 atom cluster $\mathrm{LJ}_{31}$ )), with a multitude of metastable states not much higher in energy than the global minimum, a sign of a good glass former [94]. Disconnectivity graphs for 45 and 55 atom LennardJones clusters are shown in figure 6 [86], demonstrating the high degeneracy of states near the global minimum for the glass former (BLJ 55). Heuer describes a 
method of projecting a multidimensional configuration space onto a one-dimensional potential [83], illustrated in figure 5. The energies of inherent structures were computed using extensive stochastic quenches (see section 2), and the distances between these minima $k$ in configuration space, calculated as a Euclidean distance $d\left(k_{1}, k_{2}\right)$ :

$$
\left[d\left(k_{1}, k_{2}\right)\right]^{2}=\sum_{i_{1}=1}^{N}\left(\boldsymbol{r}_{i_{1}, k_{1}}-\boldsymbol{r}_{\left[i_{2}\left(i_{1}\right), k_{2}\right]}\right)^{2}
$$

Here the difference in position of particle $i_{1}$ between basins $k_{1}$ and $k_{2}$ is computed, and summed over all particles. The notation $i_{2}\left(i_{1}\right)$ in equation (21) expresses the fact that there there is no obvious mapping from particle $i_{1}$ in basin $k_{1}$ to the same particle in basin $k_{2}$. Note that in a rigorous treatment, particle mappings between configurations and symmetries are taken into account. Reaction barriers between the inherent structures are estimated using a quartic polynomial model. This information is encoded in the transfer matrix $\tilde{V}\left(k_{1}, k_{2}\right)$, which contains the saddle point energies for the path from basin $k_{1}$ to $k_{2}$. It is then possible to construct a 1D potential with an identical transfer matrix. Using this potential and a judicious grouping of the inherent structures along the distance axis, a schematic of the PEL can be constructed. The model system in question was a 32 atom binary Lennard-Jones minimum, and the numerical predictions extracted from the projected PEL were accurate to within an order of magnitude.

\subsection{The free energy landscape}

In the potential energy landscape picture, we can indirectly recover the vibrational free energy of an inherent state by computing the local phonon density of states; however, we neglect the configurational entropy, which can be expected to have a larger contribution at low temperatures. If we want to consider higher temperatures, we must look at the free energy landscape, which includes an ensemble configurational entropy contribution at each state point, or configuration.

Although a purely thermodynamic description of glasses with the PEL can get us a long way, it is not a complete description at finite temperatures. This can be illustrated with the example of the 38atom Lennard-Jones cluster $\left(\mathrm{LJ}_{38}\right)$ [95]. It is wellcharacterised and has a double-funnel structure; the global minimum is a face-centred cubic truncated octahedron at the bottom of one funnel, and the nextlowest state is an incomplete Mackay icosahedron. Although the global minimum is thermodynamically favoured, the incomplete icosahedron has a lower free energy at approximately two-thirds of the melting temperature because the entropy is higher. Moreover, quenches from the liquid phase almost invariably end up in the icosahedron funnel since the icosahedron is closer to the polytetrahedral liquid structure. In the PEL picture, it can be said that the octahedron funnel is narrow, whereas the icosahedron funnel is broader, with a flatter basin. Close to melting, the icosahedral phase has a lower free energy. Upon entering the icosahedral funnel, the system will become trapped there even when the free energy of the global minimum phase is lower, indicating a high barrier. This is an example of a system that is pathologically difficult to globally optimise, and it gives us a great deal of insight into the differences between the PELs of crystals and glasses. Wales and Bogdan directly compare the free energy landscapes of the $\mathrm{LJ}_{55}$ system, a "structure-seeker" (i.e. crystal former) with the frustrated $\mathrm{LJ}_{38}$ system [96], from which it is possible to establish a connection between glass-forming ability and the efficiency of structure prediction algorithms. In addition to highlighting the double-funnel nature of the $\mathrm{LJ}_{38}$ system (figure 7 ), a feature on the free energy landscape points to a solid-solid phase transition from the octahedral global potential energy minimum to the icosahedral structure.

It seems, then, that glasses are governed by a complicated free energy landscape with many local minima from which the system cannot escape. Calculations on a hard sphere model suggest that at a characteristic density (which is equivalent to a characteristic temperature in the context of this model), a large number of glassy minima appear in the free energy landscape [97]. Experiments on colloidal glasses have provided the first direct evidence backing up this assertion: since the waiting time of the studied system in intermediate states varies, it can be deduced that there is a complex underlying free energy landscape, through which the system can take a variety of routes to reach an equilibrium state [98].

The cooperative rearrangements described by Adam and Gibbs arises naturally in the free energy landscape picture. A rearrangement represents a transition from one basin to another, via a free energy barrier. Crucially, the path through the barrier in phase space can be described as a "simultaneously rearranged region", or an excited state, which involves more atoms than in the equilibrium states [99]. This is visible in a glassy two-dimensional system of soft discs [100, 101], and in a hard sphere model, in which a "string-like" or looping movement of atoms appears to provide a favourable path [102].

\subsection{Discussion}

We have described some different approaches to solving the problem of glasses and the glass transition that 


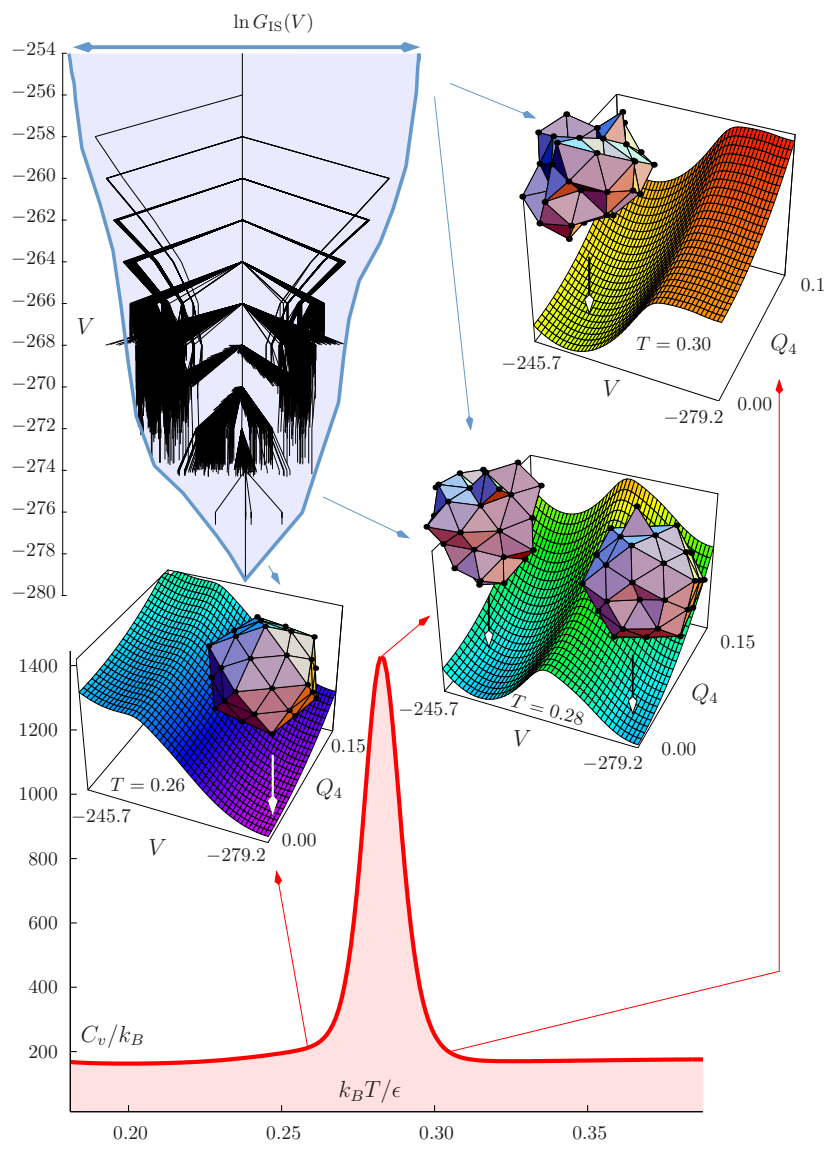

Figure 7. Disconnectivity graph (where $V$ is the potential energy), and heat capacity $\left(C_{v}\right)$ of the $\mathrm{LJ}_{38}$ cluster (reprinted with permission from J. Phys. Chem. B 110, 20765 (C)2006 American Chemical Society [96]). Insets show interesting parts of the free energy surface, as a function of potential energy and $\mathrm{Q}_{4}$, a bond-orientation order parameter. The horizontal axis of the disconnectivity graph $\ln \left(G_{\mathrm{IS}}\right)$ is the logarithm of the density of states, which is directly related to the configurational entropy. At a low temperature $(T=0.26)$, there is a single free energy minimum, corresponding to the Mackay icosahedron, while at the melting temperature temperature $(T=0.28)$, there is a double funnel structure separating the liquid-like and solid-like structures, and at a high temperature $(T=0.3)$ there is one liquid-like structure.

have different starting points, but are nonetheless closely related. RFOT is a framework that unifies three seemingly incongruous approaches: Adam-Gibbs theory, mode-coupling theory and spin-glass theory [49]. In particular, RFOT takes a similar approach to the cooperatively rearranging regions of Adam-Gibbs theory, and MCT and RFOT have identical solutions in the limit of an infinite dimensional system[70].

Although RFOT does not explicitly use the PEL formalism in its derivation, the two are closely related, since in the formulation of RFOT a large number of distinct metastable states is assumed in the glass phase, a feature that arises from analysis of the PEL of glassy systems. Moreover, the dynamic transition (analogous to the mode-coupling temperature) signifies the loss of ergodicity, as the system no longer has the kinetic energy to hop between the degenerate glassy states in the PEL picture.

One might ask whether there is a place for a seemingly qualitative approach like the PEL when there are sophisticated first-principles approaches such as MCT and RFOT available. In the words of Das [58],

'...the competition of crystallization with formation of the glassy state has not been studied from such a microscopic approach. A unified description of the amorphous and crystalline state is justified from the existing conservation laws, which hold irrespective of the nature of the thermodynamic phase.'

The "competition" between the crystalline and glass phases is one aspect that the PEL may be well suited to describing, as discussed in section 8. The PEL can be mapped for many model and real systems by calculating energies and interatomic forces using classical or quantum mechanical methods such as pair potentials or density functional theory respectively. Statisical analysis of the PEL as discussed in section 5 then allows a more quantitative analysis.

\section{Theoretical background of the potential energy landscape framework}

\subsection{Thermodynamic formalism}

Goldstein was the first to make the connection between the PEL framework and the glass transition in a somewhat heuristic way, reasoning that the potential barriers separating basins dominate the dynamical properties at low temperatures (so-called $\beta$-processes) [103]. Events occur on two timescales; high frequency vibrations within basins and Arrhenius-type activated processes corresponding to jumps over barriers between minima on a long timescale. Stillinger and Weber [79] made the first steps in a thermodynamic description of glass-formers using the topology of the PEL and the concept of the inherent structure, a term we will use interchangeably with "local minimum" and "basin". Wallace expands on this, highlighting the role of configurational entropy in the slowing of dynamics in glasses using vibrational-transitional theory [104]. This is also discussed in some depth in other review articles $[105,106]$. Working in the canonical (NVT) ensemble, with a system of $N$ non-coincident particles confined to a volume $V$, a three-dimensional system has $3 N$ spatial coordinates $\boldsymbol{r}=\left\{\boldsymbol{r}_{1} \ldots \boldsymbol{r}_{N}\right\}$. The potential energies of the set of configurations $\boldsymbol{r}, \phi(\boldsymbol{r})$, defines the PEL. It is important to note that the PEL is only dependent on atomic coordinates, and is independent of temperature. 
The crucial step is the discretisation of the continuous PEL to a set inherent structures. Relaxing the atomic positions of an arbitrary configuration such that the forces are minimised using, for example, a steepest descent or conjugate gradient algorithm, gives the local potential energy minimum in configuration space. This allows the mapping of a continuous set of coordinates $\boldsymbol{r}$ surrounding a minimum to a single minimum, $\alpha$. This mapping $M$ can be expressed as $M(\boldsymbol{r})=\alpha$, and is equivalent to an infinitely fast quench to zero temperature. $R(\alpha)$ is the set of configurations that quenches to $\alpha$, and can be described as a basin in the PEL. These basins are separated by $3 N-1$ dimensional hypersurfaces for which the mapping $M$ is undefined.

The canonical partition function for a $N$-particle system, $Z_{N}$, at temperature $T$ is,

$$
Z_{N}=\frac{1}{\lambda^{3 N} N !} \int e^{-\beta \phi(\boldsymbol{r})} d \boldsymbol{r}
$$

where $\beta=\frac{1}{k_{\mathrm{B}} T}$ and $\lambda$ is the mean thermal de Broglie wavelength. The configurational integral can be separated into distinct quench regions or basins $R(\alpha)$, so that

$$
Z_{N}=\frac{1}{\lambda^{3 N} N !} \sum_{\alpha} \int_{R(\alpha)} e^{-\beta \phi(\boldsymbol{r})} d \boldsymbol{r}
$$

However, many inherent structures are identical after particle permutation, so basins belong to an equivalence class $\frac{N !}{\sigma}$. The symmetry number $\sigma$ is unity for a system with hard boundaries, but the use of periodic boundary conditions imposes translational symmetry under which the potential is invariant, such that $\sigma=N$. Treating members of $R(\alpha)$ as equivalence classes, we have

$$
Z_{N}=\frac{1}{\lambda^{3 N}} \sum_{\alpha}^{\prime} \frac{1}{\sigma(\alpha)} \int_{R(\alpha)} e^{-\beta \phi(\boldsymbol{r})} d \boldsymbol{r}
$$

The ' here denotes that any configuration with a significant degree of crystallinity are excluded. Within any basin, the potential energy can be recast as,

$$
\phi(\boldsymbol{r})=\phi_{\alpha}+\Delta_{\alpha} \phi(\boldsymbol{r}),
$$

where $\phi_{\alpha}$ is the basin minimum and the second term is the "elevation" from that minimum. Thus,

$$
Z_{N}=\frac{1}{\lambda^{3 N}} \sum_{\alpha}^{\prime} \frac{1}{\sigma(\alpha)} e^{-\beta \phi_{\alpha}} \int_{R(\alpha)} e^{-\beta \Delta_{\alpha} \phi(\boldsymbol{r})} d \boldsymbol{r}
$$

The partition function is now separated into two distinct factors: the discrete sum over $e^{-\beta \phi_{\alpha}}$ represents the distinct temperature-independent configurations, while the integral over $e^{-\beta \Delta_{\alpha} \phi(r)}$ represents the thermal excitations of the respective configurations within their basins.

\subsection{Density of configurational states and entropy}

To make use of this formalism, we need to express it in terms of parameters that can be extracted from numerical simulations. The density of states $G(\phi)$ counts the number of basins with energy $\phi$, and can be computed by sampling the potential energy landscape using the methods detailed in section 2. It expressed using a delta function,

$$
G(\phi)=\sum_{\alpha}^{\prime} \frac{\delta\left(\phi-\phi_{\alpha}\right)}{\sigma(\alpha)}
$$

and the total partition function can be evaluated by summing over all basins:

$$
Z_{N}=\sum_{\alpha} G\left(\phi_{\alpha}\right) Z_{N, \alpha}
$$

From the thermodynamic connection, we can express the free energy per basin $f_{\alpha}$ in terms of the partition function per basin, $Z_{N, \alpha}$ :

$$
f_{\alpha}=\phi_{\alpha}-T S=-\beta \ln Z_{N, \alpha}
$$

Thus the total partition function is,

$$
Z_{N}=\sum_{\alpha} G\left(\phi_{\alpha}\right) e^{-\beta f_{\alpha}}
$$

Defining the configurational entropy as $S_{\text {conf }}=$ $k_{\mathrm{B}} \ln G(\phi)$, the partition function becomes,

$$
Z_{N}=\sum_{\alpha} e^{-\beta\left(-T S_{\mathrm{conf}}+f_{\alpha}\right)}
$$

As in section 3.1, the energy is essentially decomposed into two components: one from the configurational entropy, $T S_{\text {conf }}$, and a free energy contribution from vibrations within that basin, $f_{\alpha}$. The basins can now be considered as a continuum of levels characterised by an energy $\phi_{\alpha}$ and degeneracy $G\left(\phi_{\alpha}\right)$. When the inherent and vibrational states are in thermal equilibrium, the average configurational entropy can be computed from [107],

$$
\frac{d \bar{S}_{\mathrm{conf}}(T)}{d \bar{\phi}_{\alpha}}=\frac{1}{T}
$$

Evaluation of the integration constant combined with an extrapolation to low temperatures would then in principle allow the Kauzmann temperature $T_{K}$ to be computed as the crossover point of the entropies of the liquid and solid. This can be achieved by exploiting (29) to obtain the free energy of the liquid:

$$
\begin{aligned}
f_{\text {liquid }}(T) & =-\beta \ln Z(T) \\
& =\bar{\phi}_{\alpha}(T)-T S_{\text {conf }}\left(\bar{\phi}_{\alpha}\right)-f\left(\beta, \bar{\phi}_{\alpha}\right)
\end{aligned}
$$


Here, $\bar{\phi}_{\alpha}(T)$ is the mean inherent state energy at temperature $T$ and the vibrational free energy of the basins at $T, f\left(\beta, \bar{\phi}_{\alpha}\right)$ can be approximated from the harmonic eigenfrequency spectrum of the system calculated using the basin distribution at $T$. The configurational entropy can then be calculated as the difference between the entropy of the liquid, via thermodynamic integration, and the entropy of vibrational entropy of the solid as shown below.

\subsection{Basin free energy}

In condensed matter physics, it is common to assume that when displaced slightly from their equilibrium positions, the atoms in a crystal are subject to a quadratic potential - the harmonic approximation. The potential energy close to an inherent state can be expanded quadratically [106],

$$
V(\boldsymbol{r}) \simeq \phi_{\alpha}+\sum_{i, j, \alpha, \beta} H_{i \alpha j \beta} \delta r_{i}^{\alpha} \delta r_{j}^{\beta},
$$

where $\mathrm{H}$ is the Hessian matrix, with elements,

$$
H_{i \alpha j \beta}=\frac{\partial^{2} V(\boldsymbol{r})}{\partial r_{i}^{\alpha} \partial r_{j}^{\beta}},
$$

and $\delta_{i}^{\alpha}$ denotes a small displacement of atom $i$ in direction $\alpha$. Solving for $3 N$ eigenvalues, representing curvatures, and $3 N$ eigenvectors, representing displacement vectors, the system is equivalent to a set of $3 N$ independent harmonic oscillators with frequency $\omega_{j}$. The partition function of a single basin can then be expressed,

$$
Z_{\alpha}=e^{-\beta \phi_{\alpha}} \prod_{j=1}^{3 N} \frac{1}{\beta \hbar \omega_{j}\left(\phi_{\alpha}\right)}
$$

Averaging over all basins, this becomes,

$$
Z_{N}=e^{-\beta \phi_{\alpha}}\left\langle e^{-\sum_{j=1}^{3 N} \ln \left[\beta \hbar \omega_{j}\left(\phi_{\alpha}\right)\right]}\right\rangle_{\alpha}
$$

Thus the basin free energy $f_{\alpha}$ in the harmonic approximation can be written,

$$
\begin{aligned}
-\beta f_{\alpha} & =-\beta \phi_{\alpha}-\beta f_{\mathrm{vib}} \\
& =-\beta \phi_{\alpha}-\ln \left\langle e^{-\sum_{j=1}^{3 N} \ln \left[\beta \hbar \omega_{j}\left(\phi_{\alpha}\right)\right]}\right\rangle_{\alpha}
\end{aligned}
$$

The vibrational free energy $f_{\text {vib }}$ is often approximated to,

$$
\beta f_{\mathrm{vib}}=-\left\langle\sum_{j=1}^{3 N} \ln \left[\beta \hbar \omega_{j}\left(\phi_{\alpha}\right)\right]\right\rangle_{\alpha}
$$

Simulations on a binary Lennard-Jones model glassforming system suggest that the harmonic approximation is appropriate below the glass transition temperature, in the slow dynamics regime [108]. These results also seem to indicate a transition from a harmonic regime below the crossover temperature to an anharmonic regime above it, but it is unclear whether this is true in general.

\section{Schemes for sampling the PEL}

One of the principal difficulties in modelling amorphous solids is the construction of microscopic structural models that are representative of the physical reality. Amorphous materials are more difficult to characterise experimentally, since structural disorder causes broadening of spectral features that could be used to identify the atomic structure. In many cases, it is unclear whether a glass is disordered on the atomic level, or whether it has some form of superatomic unit, or shows signs of medium-range (quasicrystal-like) order. Our aims are twofold: firstly, we want to map out the PEL such that we can determine whether the system is a glass- or crystal-former, and secondly, we need to develop physically representative models that can be used to perform computer experiments on specific materials. In this section, we discuss several methods of achieving these goals.

\subsection{Reverse Monte Carlo (RMC)}

The reverse Monte Carlo technique employs experimental data in the form of structure factors, the Fourier transform of the radial distribution function, to create a consistent structural model. The pair distribution function of a random configuration of experimental density is used to compute the structure factor, and the difference between the model and experimental structure factors is used to define a generalised cost function. A single atom is moved in the model configuration, and the move accepted or rejected according to a probability distribution determined by the cost function. This algorithm will converge the model structure factor to the experimental structure factor [109]. RMC does not generate a unique structure, but a set of structures determined by constraints imposed by experimental data. This may include structures with unphysical coordinations, but which are otherwise consistent with the experimental data. In extension to this method, experimentally constrained molecular relaxation (ECMR), ab initio simulation methods are used to obtain a model that is consistent between the experimentally generated RMC data and the simulated system [110].

\subsection{Molecular dynamics: aging versus equilibrium}

When a liquid is cooled, it falls out of equilibrium at the glass transition, i.e. the dynamics become so slow that it is impossible to equilibrate; beyond this point the structure relaxes so slowly that it is extremely difficult to determine the functional form of the relaxation on an experimental timescale. This experimentally imperceptible structural relaxation is known as "aging." Cathedral glass panes which are 
thicker at the bottom are a common and most probably apocryphal example of glass flow on long timescales; in fact, it is highly unlikely that such glasses age on a human timescale [111]. Fossilised amber, aged for 20 million years presents a more convincing case for aging: it has a density $2.1 \%$ higher than thermally rejuvenated amber, and provides evidence that the relaxation may take a form that is not described well by the most widely used models, including VFT [112]. In fact, analysis of data for glass forming liquids has largely discredited VFT [113], and the best fit for relaxation in amber is the parabolic law model of Elmastad et al. $[114,115]$. This not only brings into question the validity of the VFT law, but it also throws doubt on dependent theoretical paradigms such as the AdamGibbs formalism, and the existence of the Kauzmann temperature, which arises naturally from the VFT form.

Molecular dynamics in principle allows the computation of time-dependent properties of a system, and should be the best method of modelling the liquid-glass transition. However, while it would be of great benefit to be able to model the evolution of the system in real time, given the structural disorder, it is prohibitively expensive to run simulations for long enough to capture the slow dynamics of a glass. The time step must be chosen to be smaller than the shortest vibrational time period of the system, i.e. of the order of femtoseconds, which severely limits the potential length of simulations to milliseconds for classical molecular dynamics and nanoseconds for $a b$ initio simulations. Longer timescales can be achieved using accelerated or coarse-grained molecular dynamics, but this also adds to the uncertainties.

Kob and Andersen [28] performed molecular dynamics simulations on a binary Lennard-Jones mixture with a 4:1 ratio of atomic species and forcefield parameters, both chosen to avoid crystallisation, the 80:20 BLJ system. They observe that in many glass-transition simulations, certain thermodynamic quantities such as the potential energy per particle display a discontinuity with respect to temperature, denoted $T_{d}$. This simulation temperature is often much higher than the laboratory glass transition temperature $T_{g}$. In fact, $T_{d}$ is indicative of the system falling out of equilibrium, with an equilibration timescale greater than that of the simulation, i.e. a glass transition. $T_{d}$ depends on the cooling rate and the thermal history of the system. Moreover, monitoring the system via simple time-dependent variables such as energy or pressure might suggest that the system is in equilibrium, as their values stabilise; a more careful examination using a two-point correlation function such as non-equilibrium generalisation of the incoherent scattering function $C_{q}$ suggests an aging process [116].

$$
C_{q}\left(t_{w}, t_{w}+t\right)=\frac{1}{N}\left\langle\sum_{i=1}^{N} e^{i \boldsymbol{q} \cdot\left[\boldsymbol{r}_{i}\left(t_{w}+t\right)-\boldsymbol{r}_{i}\left(t_{w}\right)\right]}\right\rangle
$$

Here, $t_{w}$ is the time since the quench at $t=0$, and $\boldsymbol{q}$ is a wave vector.

The van Hove self correlation function describes the probability of finding a particle at distance $r$ from its original $(t=0)$ location at time $t$ :

$$
G_{s}(r, t)=\frac{1}{N} \sum_{i=1}^{N}\left\langle\delta\left(\left|\boldsymbol{r}_{i}(t)-\boldsymbol{r}(0)\right|-r\right)\right\rangle,
$$

and is essentially a time-dependent radial distribution function. Its spatial Fourier transform is the self intermediate scattering function $F_{s}(k, t)$ (identical to the dynamical structure factor described in section 2.1). Using the same Lennard-Jones binary mixture Sastry et al. were able to associate the onset of the non-exponential relaxation characteristic of the glass transition with a well-defined temperature which corresponds to the trapping of the system in the deepest accessible basin on the PEL [117]. Defining the relaxation time $\tau$ as the time when $F_{s}(k, t)$ reaches a value of $1 / e$, they identify three distinct temperature regimes: the first is the high temperature regime, in which the system has sufficient kinetic energy to surmount activation barriers and explore configuration space, and $F_{s}$ has an exponential form. As the temperature decreases to the "landscape-influenced" regime, a stretched exponential form $F_{s}(k, t)=$ $\exp \left[(-t / \tau(T))^{\beta(T)}\right]$ (where $0<\beta<1$ ) becomes a better fit. In the "landscape-dominated", which corresponds to just above $T_{\mathrm{MCT}}$, small secondary peaks are observed in $G_{s}$, implying the occurrence of rare jumps by particles over a distance of about the interparticle separation. Thus at high temperatures, the system explores parts of the PEL with low minima between basins, and at temperatures close to $T_{\mathrm{MCT}}$, minima separated by considerably higher barriers are explored. In the PEL picture, the dynamics become dominated by the landscape at a crossover temperature close to $T_{\mathrm{MCT}}[118]$.

Most studies have the explicit aim of examining equilibrium properties, but one in particular uses non-equilibrium molecular dynamics to describe the aging process in a glass [119], notably demonstrating using the 80:20 BLJ system, that non-equilibrium dynamics are dominated by the connectivity of the basins in configuration space, and by extension, the height of the barriers separating them. The aging of the system is shown to be determined by a time-dependent effective temperature $T_{e}$, defined by inverting the time-dependence of the temperaturedependent basin energy $\phi_{\alpha}(T)$ to get $T_{e}\left(\phi_{\alpha}\right)$. As 
the system ages, it locates deeper basins, reducing its value of $T_{e}$, until it reaches the point when the barrier heights become of the order of $k_{\mathrm{B}} T$. At this point the exploration of configuration space changes from moving along unstable modes to an activated process in which barriers must be surmounted. This activated process is considerably less efficient for exploring the PEL. Aging can thus be considered the exploration of progressively deeper basins in the PEL picture; this concept has been extended by considering the shape of the basins during aging, and its dependence on the temperature to which the system is quenched. Specifically, for the $80 / 20$ binary Lennard-Jones system, basins of a higher curvature (and therefore higher vibrational frequency) are explored below the $T_{\text {MCT }}$ [120]. In the case of supercooled molecular liquids, it has been shown that rotations occur over a longer timescale than translations, and the time taken for rotational correlations to reach equilibrium is more strongly temperature-dependent than for translational correlations [121]. Lempesis et al. introduce a variation on the familiar disconnectivity graph, the temporal disconnectivity graph. A time (or equivalently temperature) axis is added to a disconnectivity graph to turn it into a 3D plot, and demonstrate the effects of aging on inter-basin communication [122].

Tsalikis et al. also present results describing three distinct temperature regimes, but these are of a different nature. High above $T_{g}$, the sampling of the PEL is ergodic, and is relatively computationally inexpensive. In the second regime, in the vicinity of $T_{g}$, the sampling is inefficient, and in the third, below $T_{g}$, the system is essentially trapped indefinitely [123]. The most interesting conclusion of this work is that the nature of the glass formed is strongly dependent on the time spent by the system in the second temperature regime. They conclude that the cooling rate, particularly when it is conducted in a stepwise manner as in most molecular dynamics simulations, is not sufficient to define the resulting glass, since the time spent at each temperature has an extremely non-linear effect.

La Nave et al. used instantaneous normal mode analysis (see section 5.3) to estimate the fraction of diffusive directions $f_{\text {diff }}$ of equilibrated representative configurations of silica at temperatures both above and below the crossover [124]. A direction on the PEL is considered to be diffusive if, when displaced in that direction, the system moves into a different basin, and $f_{\text {diff }}$ is defined as the ensemble average $\left\langle N_{u} / 3 N\right\rangle$, where $N_{u}$ is the number of directions with negative eigenmodes excluding those displaying signs of anharmonicity. It quantifies the amount of time the system spends on the ridges of the PEL between basins. $f_{\text {diff }}$ is seen to decrease as the temperature decreases, and vanishes at $T_{\mathrm{MCT}}$, implying that the number of directions leading to a different basin decrease with temperature, which makes sense given that below $T_{K}$, the system is trapped in a single basin. Moreover, for silica, they find the direct relationship $S_{\text {conf }} \propto$ $\log f_{\text {diff }}$ between the configurational entropy $S_{\text {conf }}$, which essentially enumerates the number of distinct basins explored by the system, and $f_{\text {diff. }}$. This seems to be system-independent, and holds for both strong and fragile dynamics.

Similar studies lead to some even stronger conclusions. Saddle points on the PEL are classified according to the number of imaginary modes in the Hessian matrix, $n_{s}(T)$, and the energy of that configuration, $e_{s}(T)$. At $T_{\mathrm{MCT}}, n_{s}$ vanishes, and at higher temperatures it increases with a well defined functional form. This makes $n_{s}$ a good choice of parameter for describing the dynamics of supercooled liquids, and it can be used to calculate $T_{\mathrm{MCT}}$ without using mode-coupling theory. In addition, the results suggest that above $T_{\mathrm{MCT}}$, the dynamics are entropy driven rather than activated, with the system following a path through saddle points with a progressively decreasing value of $n_{s}[125,126]$. This finding supports the validity of the "potential energy landscape ensemble" paradigm [127, 128] (see section 5.5). Since the Hessians are known at all saddle points, it is possible to compute the partition function at the saddles, which can be used in transition state theory [126].

"Melt-quench" molecular dynamics is the traditional method of generating structural models of glasses [129-131]. A crystalline phase simulated using molecular dynamics is heated to well over its melting temperature, and the resulting liquid is equilibrated. The temperature is then gradually lowered in a stepwise manner, equilibrating at each temperature increment, until the glass transition is reached and the structure will no longer equilibrate on a reasonable timescale. However, the ability of this method to generate representative amorphous structures is debatable. In principle, the instantaneous structure of a liquid is indistinguishable from that of a glass; however, the use of a single trajectory to generate many quenched configurations may introduce unwanted correlations. Moreover, glasses are formed at low cooling rates in practice, and the simulation timescales required for such low cooling rates are impractical for both classical and $a b$ initio molecular dynamics. Whilst it is possible to simulate a low cooling rate using small temperature drops followed by periods of equilibration, the cooling rate is still orders of magnitude higher than in practice, and it is unclear whether the resulting configurations represent those found experimentally. A further caveat is that this method is only 
useful if the local structure of the liquid phase is similar to that of the amorphous solid phase; this is true in the case of $\mathrm{SiO}_{2}$ and $\mathrm{GeSe}_{2}$, but does not hold for amorphous silicon, which is tetrahedrally coordinated as an amorphous solid, but sixfold coordinated as a liquid [132]. This discrepancy does not appear in the structure factor of amorphous Si generated by meltquench, which tends to contain the wrong density of coordination defects regardless of the level of theory used in the potential [133]. This serves as a caution against careless application of melt-quench molecular dynamics.

\subsection{Density of states Monte Carlo}

Monte Carlo sampling allows a time-independent sampling of the PEL. This has the advantage of sidestepping the slow dynamics and equilibration issues. Yan et al. perform Monte Carlo simulations in the "multimicrocanonical ensemble," in which the particle number and volume are constant, but the total energy is allowed to fluctuate over a range [134]. A random particle is displaced slightly, and the move is accepted with a probability determined by the difference in energy between the states, as per the standard Metropolis Monte Carlo algorithm. Since the density of states is not known a priori, it is computed iteratively. The heat capacity can then be computed from the density of states. The results of simulations on a 50:50 binary LennardJones mixture show the peak in heat capacity and sharp drop in inherent structure energy as a function of temperature characteristic of a glass transition. Crucially, the results conflict with direct molecular dynamics simulations [56] which used a power law to extrapolate the entropy of the liquid to low temperatures and determine the intersection with the entropy of the disordered solid. The Monte Carlo simulations suggests that the power law does not hold at low temperatures, and that there is no such crossover (Kauzmann) temperature.

\subsection{Continuous Random Networks (CRN)}

Continuous random networks were originally conceived to generate structural models for amorphous $\mathrm{Si}$ and Ge, by preserving local order, while randomising bond lengths and angles. Starting from a supercell of a periodic diamond structure, bonds are locally rearranged by "rewiring" bonds which are modelled with a naïve "spring" potential, maintaining the average coordination, but introducing five- and sevenrings in addiction to the six- and eight-rings in the crystalline structure. The structure is then allowed to relax to within the constraints of the new nearestneighbour list. This is repeated until the structure is deemed to be sufficiently random [135].

Variations on this technique involve starting from a crystalline melt or a random four-coordinated configuration, and using a Metropolis-type algorithm to accept or reject moves [136]. This method has been applied successfully in modelling, for example, amorphous silicon [137] and silica [138], but its application is limited to strong liquids, i.e. glassforming materials where the atoms have a very definite coordination number and form distinct covalent bonds.

\subsection{Stochastic Quenching (SQ)}

The SQ method bears some similarity to meltquench molecular dynamics, but instead of rapidly quenching snapshot configurations from a potentially expensive and time consuming equilibrium molecular dynamics simulation of the respective liquid phase, a "sensible" random initial configuration is quenched infinitely fast. 'Constrained stochastic' configurations are generated by randomly distributing atoms in a cubic box with periodic boundary conditions, subject to the constraint that no two atoms may be too close, since this may interfere with electronic structure optimisation, and cause instability in geometry optimisation algorithms. Each configuration is optimized to the potential energy local minimum [139]. In materials where the characteristic length scale is unknown, a similar process can be performed, randomly placing superatomic units in the unit cell instead of atoms; this may be appropriate when the crystal structure consists of easily identifiable superatomic units.

SQ is thought to be valid because it has been demonstrated that, for a monatomic liquid, the vast majority of all structures are random, and the normal mode frequencies are essentially the same for all random structures. The distribution of frequencies takes the form of a Gaussian distribution with a variance that is independent of temperature [104, 140]. Thus any randomly generated structure should in principle have the same structural properties.

SQ has been shown to accurately reproduce structural and chemical features of even complex amorphous systems such as $\mathrm{ZrSiC}$. Disagreements between theory and experiment have been attributed to the fact that SQ is effectively a quench with an infinitely fast cooling rate [141]. However, this agreement may be a result of insufficient analytical rigour. In the case of $\mathrm{GeSe}_{4}$ glasses, the structure factors for stochastic quenched structures are very similar to those for structures formed from a new melt quench technique in which the Ge atoms are localised in either a sphere or a slab embedded in amorphous Se. The NMR spectra tell a different story: when compared with experimental NMR spectra, 
stochastic quenching leads to an overly homogeneous structure, suggesting that the glass may be comprised of superatomic units that only form during a melt [142]. If it is possible to identify these superatomic units, it may be more accurate to perform stochastic quenching by randomly placing them in the cell rather than single atoms.

This method is very similar to ab initio random structure searching (AIRSS) [84, 143], in which ensembles of sensible stochastic configurations in considerably smaller cells are quenched at zero temperature to determine crystal structures. The key difference in AIRSS is the exploration of the enthalpy landscape rather than the potential energy landscape as in the SQ method.

\subsection{Activation-Relaxation Technique (ART)}

ART [144, 145] employs a completely different method of exploring the PEL by taking into account the activation barriers between basins. Molecular dynamics simulations are incapable of sampling the configuration space since significant structural changes happen on a timescale that is considerably longer than experiments, let alone simulation. In a glass, the system is essentially confined to vibrational motion within a basin.

Starting from a quenched configuration at a basin minimum, a single atom is displaced slightly such that the total force is non-zero. The configuration is then pushed to a saddle point by following the PEL in a direction of increasing force (essentially the inverse of a geometry optimisation); this may involve the motion of any number of atoms. Rather than using forces computed from the full dynamical Hessian matrix as in a phonon calculation, a "redefined" force $\boldsymbol{G}$ is used.

$$
\boldsymbol{G}=\boldsymbol{F}-\left[1-\frac{\alpha}{1+\Delta x}\right](\boldsymbol{F} \cdot \Delta \boldsymbol{x}) \Delta \boldsymbol{x}
$$

Here, $\boldsymbol{F}$ is a $3 N$-dimensional force vector derived from the gradient of the potential energy, $\Delta \boldsymbol{x}$ is displacement vector of the single atom from the local minimum and $\alpha$ is a parameter determining the rate of ascent to the saddle point.

From the saddle point, the system is quenched into a different local minimum. The net result is the system hops from one basin to another. This process is repeated to explore the potential energy landscape. This method has the advantage that its efforts are concentrated in the tiny region of configuration space that is accessible (since most of configuration space is comprised of unphysical structures); on the other hand, it could be argued that a bias is introduced by limiting the volume of configuration space explored in this way. ART is good at locating transitions between metabasins (funnels) compared with molecular dynamics, in which transitions are weighted heavily by a Boltzmann factor [146].

\subsection{Discussion}

There are several methods for generating structural models of glasses, but all have advantages and disadvantages. Melt-quench molecular dynamics is by far the most widely used in spite of the fact that there are many reasons not to blindly trust it. In the cases where the liquid and crystalline phases have different first-neighbour coordination numbers, for example amorphous silicon, it fails miserably [133]. The fact that there is a time-dependence between configurations may introduce unwanted correlations, and it is impossible to reach cooling rates close to experiments due to the compuatational expense. Moreover, it is only physically representative of one method of producing glasses (cooling a liquid), and not vapour deposition, which is a highly non-equilibrium process.

The CRN method intriguingly works well situations in which melt-quench molecular dynamics invariably fails (amorphous Si). This leads to the suggestion that bond rewiring may be representative of the underlying physics — indeed, it appears that bond switching events actually happen frequently over long timescales [145]. On the other hand, CRN is clearly inappropriate for fragile glass-formers such as bulk metallic glasses, where there are no strong directional bonds.

When compared with stochastic quenching, meltquench molecular dynamics consistently generates lower energy configurations; however, unlike in crystal structure prediction, we are not searching for the thermodynamic ground state. In fact, experiments comparing a quenched glass with a ultrastable vapourdeposited glass for the metallic glass $\mathrm{Zr}_{65} \mathrm{Cu}_{27.5} \mathrm{Al}_{7.5}$ demonstrate that the ultrastable glass has a $T_{g}$ that is $11 \mathrm{~K}$ higher, but has a considerably higher enthalpy [9]. This is a point that is rarely considered and remains an open question; similarly, when using stochastic quenching, it raises the question of how to choose the most representative structure from an ensemble.

\section{Theoretical characterisation of glassy structures}

In this section, we shift the emphasis from generating structural models of glasses to analysing them. Since these amorphous configurations lack the order of crystals, we need rigorous statistical methods for describing their structure and dynamics. 


\subsection{Structure and packing}

It is commonly argued in the literature that it is impossible to describe the glass transition using only static structural models, and that it is necessary to describe the dynamic heterogeneity of the system, i.e. the inhomogeneous distribution of frozen and cooperatively mobile regions as in RFOT (section 2.2). Instead of asking which aspects of the structure give rise to the dynamic heterogeneity, a question that has yet to be answered, Widmer-Cooper et al. ask which aspects of the dynamic heterogeneity arise from the structure [147]. In a two-dimensional system of soft discs, they find that while the initial configuration of a dynamical system does not directly determine the locations of diffusive regions, it does determine the propensity of particles to undergo large displacements, where the propensity is defined as the ensemble average of mean-squared displacements of a particle.

Traditionally, microscopic models of amorphous solids have been based on random arrangements of hard-sphere atoms, the dense random-packed model. However, the microscopic structure of a glass locally resembles the crystalline state of the same composition, with a non-periodic packing of the same polyhedral building blocks [148]. Attempts to describe liquids as disordered crystals and dense gases have both failed, suggesting that a liquid is a well-defined phase. This is evidenced by the fact that simple liquids can be supercooled well below their melting point without crystallising proving the existence of interphase energy in the form of the Gibbs free energy of crystallisation, and therefore the difference in liquid and solid structure [149].

A notable feature of metallic glasses is that they have a high density relative to the crystalline phase, up to $99.5 \%$ of the crystalline density in the case of the best glass forming systems. This highlights the importance of efficient atomic packing, which can be best achieved with efficiently packing solutecentred clusters such as tetrahedra and octahedra. The relative sizes of the constituent atoms are critical in this regard [150]. Two ranges of order are present in this scenario: short range order, namely ordering in the first coordination shell of the particles, and medium-range order, the ordering between the notional clusters resulting from the short range ordering. Such a paradigm requires efficient space-filling on two levels, and it has been shown that icosahedral medium-range ordering is a general feature regardless of the type of short range ordering present [151].

Frank was the first to suggest that atoms in a liquid cluster in the form of an atom-centred deformable icosahedra [152]. It is impossible to fill space with regular tetrahedra, so any attempt to fill space must involve distorted tetrahedra which introduces strain into the system. This accounts for the larger strain energies in liquids. Considering clusters relaxes the space-filling criterion, so smaller clusters favour liquid-like polyhedral packings [153]. The most favourable polytetrahedral packing consists of icosahedra, which enhances the short range packing (in contrast with the face-centred cubic and hexagonal close packed crystal systems, which improve the long range packing) [149]. This goes some way towards explaining the resistance of supercooled liquids to crystallisation - it requires a substantial atomic rearrangement. Moreover, five-fold symmetry is forbidden in crystals and is a barrier to crystallisation, but has been observed, for example, in simple monatomic liquid lead [154]. Icosahedral ordering has also been demonstrated from a model of binary alloy, amorphous Ni-Ag, constructed using reverse Monte Carlo [155]. Experimental studies have also reported icosahedral clustering in quaternary bulk metallic glasses, where the addition of a fourth species greatly increases glass-forming ability — in such systems, the ratio of atomic sizes between solute and solvent is the most important factor [156]. Whilst there is a broad spectrum of polyhedra present in metallic glasses, the dominant species varies with the atomic size ratio [151]. There is evidence that the proportion of icosahedral structures in a glass is an indicator of glass-forming ability. For example, lower cooling rates result in configurations containing more full icosahedra [157], and the glass-forming alloy $\mathrm{Cu}_{45} \mathrm{Zr}_{45} \mathrm{Ag}_{10}$ has a higher proportion of icosahedron-like clusters [158]. However, the characteristics of such glasses are not only dependent on topology, but are also highly dependent on the chemistry of the constituent elements. Although density changes in amorphous solids experimentally correlate with glass forming ability, existing dense packing models have failed to predict the glass forming compositions of the CuZr system, for example [159].

Based on X-ray and neutron scattering experiments, Ma et al. propose a fractal packing scheme for metallic glasses [160]. The polyhedral units of a quasicrystal cannot tessellate to fill space due to symmetries (such as five-fold) forbidden in crystalline lattices, but instead tessellate to form a structure without translational symmetry, and are fractals with a dimension of 2.72. Similarly, the icosahedral solute-centred clusters that form metallic glasses cannot develop any order beyond a few cluster lengths, i.e. medium-range order. This results in a fractal network with a dimensionality of 2.31 , lower than that of a quasicrystal since space-filling is more difficult in a glass.

The solute-centred quasi-equivalent cluster model is distinct from the simpler solute-centred model detailed above in that there is no orientational order between the clusters, so that the solvent atoms occupy 
random positions [161]. It is capable of predicting the number of solute atoms in the first coordination shell of a particular solvent atom, allowing the calculation of atomic concentrations in bulk metallic glasses. There is also recent experimental evidence to support this model [162].

These solute centred clusters are often defined using Voronoi tessellation analysis [163, 164]. The Voronoi cell or polyhedron of an atom contains the set of points that are closer to that atom than any other, and is analogous to the Wigner-Seitz cell in crystalline structures. The coordination of the central atom is described by the Voronoi index $\left\langle n_{3}, n_{4}, n_{5}, n_{6}, \ldots\right\rangle$, where $n_{i}$ is the number of $i$-edged polyhedra and $\sum_{i} n_{i}$ is the coordination number [2]. Voronoi tessellation analysis provides a rigorous definition of the free volume of an atom, i.e. the space into which an atom is capable of moving, namely the volume of its Voronoi polyhedron. By comparing the volumes of Voronoi polyhedra between liquids, glasses and crystals, it has been shown that it is not possible to distinguish a static snapshot of a crystal from a liquid or amorphous solid using distributions of free volume [165, 166]. Modelling a 2D system of soft discs, Widmer-Cooper and Harrowell conclude that the mobility of a particle as characterised by its DebyeWaller factor (essentially the mean-squared deviation of a particle from its equilibrium position, averaged over all particles) is not dependent on its geometrical free volume, since particles with a high free volume are uncorrelated with particles with a high DebyeWaller factor - free volume is a strictly single-particle property, and as such fails to capture the cooperative dynamics over even short timescales. [167].

Free volume models are an attractive idea, supporting the notion that the critical point in the glass transition is when the structure changes to such a degree that there is no longer enough free volume for atoms or molecules to percolate through the amorphous matrix. Free volume is just one element of the description of percolation, but in order to distinguish between phases, one also needs to take collective motion into account. It now seems that past successes of this model [168] may have resulted from an inconsistent definition of free volume.

\subsection{Density of states}

The configurational density of states (DOS) $G\left(E_{\mathrm{IS}}\right)$ enumerates the frequency of local minima (inherent states) of energy $E_{\mathrm{IS}}$. The canonical partition function can be written as a function of the density of states, $G\left(E_{\mathrm{IS}}\right)[169]$ :

$$
Z(T)=\sum_{\mathrm{IS}} G\left(E_{\mathrm{IS}}\right) \exp \left(-\frac{\left\langle F_{\mathrm{IS}}\right\rangle}{k_{B} T}\right)
$$

and $G(E)$ can be written as a function of the configurational entropy $S_{\text {conf }}(E)[29]$ :

$$
G\left(E_{\mathrm{IS}}\right)=\exp \left(\frac{S_{\mathrm{conf}}\left(E_{\mathrm{IS}}\right)}{k_{B}}\right),
$$

and it should be noted that this excludes the vibrational contribution from all minima. The density of states can be constructed by sampling the PEL and constructing a histogram of inherent state energies. $G\left(E_{\mathrm{IS}}\right)$ is commonly approximated as a Gaussian probability distribution, according to the random energy model [169-171]:

$$
P\left(E_{\mathrm{IS}}\right)=\frac{\exp (\alpha N)}{\sqrt{2 \pi \sigma^{2}}} \exp \left(-\frac{E_{\mathrm{IS}}^{2}}{2 \sigma^{2}}\right) \delta E,
$$

where $P\left(E_{\mathrm{IS}}\right)$ is the probability of finding an inherent structure in the range $E_{\mathrm{IS}} \rightarrow E_{\mathrm{IS}}+\delta E, \exp (\alpha N)$ is an estimate of the total number of basins as in equation (20), and $\sigma$ is the Gaussian width. This approximation does not hold for low energies, since the density of states of a glass former is characterised by a fat tail, such that there is a non-trivial probability of finding a structure with an energy close to the crystalline ground state. This feature is not limited to amorphous systems, however [172], but it may be possible to distinguish a glass-forming PEL from a crystal forming PEL from the functional form of the low-energy tail.

A comparison between a fragile liquid, a binary Lennard-Jones mixture, and a strong liquid, $\mathrm{SiO}_{2}$, shows a clear distinction in their respective DOS: $\mathrm{SiO}_{2}$ has a well defined cutoff energy, below which the density of states suddenly falls to zero, while the BLJ system has a smooth $G(E)$ that falls to zero as a Gaussian [173].

Whilst it is impossible to directly sample the total number of local minima in a configuration space, a recent study on hard spheres has demonstrated that it is possible to estimate it by calculating the hypervolumes of basin minima [174]. Given the basin volume, it is possible to calculate the entropy of the distinct minima, and thus the number of distinct states.

\subsection{Normal mode analysis}

The application of normal mode analysis to liquids and glasses allows access to a wealth of thermodynamic information, including the barrier-crossing rate and hence the diffusion constants. Most significantly, it presents the possibility of describing the dynamic heterogeneity of glasses from a static picture. Simulations on a $2 \mathrm{D}$ system of soft discs suggest that the locations of regions of irreversible structural reorganisation (i.e. diffusion) are strongly correlated with the 
positions of the local and non-local low frequency normal modes of the initial configuration. Furthermore, positions of these low frequency modes persists over changes in inherent structure [175]. These diffusive regions may correspond to the dynamic heterogeneities that are thought to be necessary to describe glasses in random first-order transition theory [22].

Stillinger and Weber used local minima to describe the topology of the PEL, essentially ignoring the saddles that separate them. One can also describe the PEL in terms of the density of saddles. The calculation of the normal modes of a static snapshot of a dynamic trajectory is known as instantaneous normal mode analysis (INM). In contrast to a crystal structure, liquids and glasses possess both stable (real) and unstable (imaginary) modes, such that the total density of vibrational states can be decomposed into a sum of stable $\left(\rho_{s}\right)$ and unstable $\left(\rho_{u}\right)$ modes [176]. These unstable modes are thought to be transition pathways between basins. Zwanzig proposed a relationship between the basin-hopping frequency $\omega_{h}$ and self-diffusion constant $D(T)$ [177] based on the PEL formalism, and Keyes related the hopping frequency to quantities measurable in numerical simulations, namely normal mode frequencies, and the subset of normal mode frequencies that are unstable [178]. We define the frequency of a one-dimensional configuration escaping a minimum of frequency $\omega_{m}$ via a barrier of frequency $\omega$ as $\omega_{e}$ :

$$
\omega_{e}=f\left(\omega, \omega_{m}\right) e^{-\beta E_{A}}
$$

where $f\left(\omega, \omega_{m}\right)$ is a rate constant. Generalising this to an escape from a minimum on a $3 N$-dimensional PEL, we need to take into account the cross-section of the $3 N-1$-dimensional barrier $v(\omega)$ and the volume of the minimum $v_{m}$.

$$
\omega_{e}=\left(\frac{\omega}{\omega_{m}}\right)\left(\frac{v(\omega)}{v_{m}}\right) f\left(\omega, \omega_{m}\right) e^{-\beta E_{A}}
$$

Then the hopping frequency $\omega_{h}$ is the integral of this expression over all minima and saddles.

$$
\omega_{h}=\int d \omega\left(\frac{\omega}{\omega_{m}}\right)\left(\frac{v(\omega)}{v_{m}}\right) f\left(\omega, \omega_{m}\right) \operatorname{sn}(\omega)\left\langle e^{-\beta E_{A}}\right\rangle_{\omega}
$$

The $\operatorname{sn}(\omega)$ term integrates to the number of saddles $s$ connecting the minima. Using assumptions similar to the Adam-Gibbs law, the density of unstable vibrational states $\rho_{u}$ can be expressed,

$$
\begin{aligned}
\left\langle\rho_{u}(\omega ; T)\right\rangle & =\left(\frac{\alpha}{3 z}\right) S(\omega ; T)\left[1+\int d \omega S(\omega ; T)\right]^{-1} \\
S(\omega ; T) & =\left(\frac{s}{m}\right)\left(\frac{v(\omega)}{v_{m}}\right) n(\omega)\left\langle e^{-\beta E_{A}}\right\rangle_{\omega}
\end{aligned}
$$

Here $\alpha / 3 z$ is the fraction of the $3 z$ directions with downward curvature corresponding to an unstable mode. The similarity in pre-factors suggests a relationship between $\omega_{h}$ and the $\rho_{u}$, which can be evaluated by fitting a numerically computed density of unstable states to a functional form; the troublesome saddle point terms are also eliminated in this way. However, it should be noted that there may be imaginary modes in a crystal at finite temperature, i.e. non-diffusive unstable modes, and by extension these exist in liquids and glasses. The problem with INM is determining which of these unstable modes are diffusive [179].

\subsection{Activated dynamics}

Aging is a phenomenon that occurs on a timescale orders of magnitude beyond what is accessible from molecular dynamics simulation; diffusive processes that occur beyond experimental timescales allow a glassy system to find lower thermodynamics states. In the PEL paradigm, diffusion in a supercooled liquid can be described in terms of cage-breaking (i.e. a particle breaking out of a cage formed of its nearest neighbours). Productive, or non-reversed, cage-breaks correspond to transitions between metabasins, whereas non-cage-breaking process correspond to non-diffusive processes occurring within a metabasin [180]. Below the glass transition, the former are strongly suppressed, resulting in super-Arrhenius relaxation. This issue can be circumvented by considering trajectories through configuration space as discrete transitions between local minima. The problem of dynamics on the PEL can be greatly simplified by considering a transition between two connected local minima as a two state transition problem. When there is an energy barrier of height $E_{A}$ (an activated process), the statistics of transitions between microstates can be modelled using (for example) an Arrhenius-type rate equation, where the transition rate is proportional to $e^{-\frac{E_{A}}{k T}}[181]$.

We now turn to how this enables dynamical simulations to be coarse grained and therefore performed on larger systems. Instead of using brute force molecular dynamics, the slow dynamics of a glass can be modelled as a Poisson process of elementary transitions between local minima, controlled by a rate parameter $\lambda$ quantifying the expected number of events per unit time [123]. Such a (Markovian) process has no "memory" of the previous states of the system, much like radioactive decay. An "event" is defined as the transition of the configuration from one basin to a neighbouring basin of higher energy. The cumulative probability $P(t)$ is of the form,

$$
P(t)= \begin{cases}1-e^{-\lambda\left(t-t_{0}\right)} & t>t_{0} \\ 0 & t \leq t_{0}\end{cases}
$$


where $t_{0}$ is the time of the last event. Then the gradient $\lambda$ is,

$$
\lambda=\frac{n-r_{l}}{\sum_{k=r_{1}+1}^{n}\left(t_{k}-t_{l}\right)},
$$

where $n$ is the total number of transitions, $t_{k}$ is the residence time of transition $k$ and $r_{l}$ is the first data point to consider when calculating the slope (since the Poisson approximation does not hold at short times). Tsalikis et al. [182] validated this method by comparing the results of the Poisson process with the mean squared deviation for the molecular dynamics simulations used to determine the rate constant. This approach recovers the twotimescale behaviour that typifies glassy systems: short timescale transitions occur within metabasins, and long timescale transitions occur between metabasins. Moreover, these transitions can be related with the separation of the local minima in configuration space as parameterised by the Euclidean norm: intrametabasin and intermetabasin transitions have different characteristic "lengths". These long distance transitions correspond to collective rearrangements, whereas short distance transitions correspond to isolated cage-breaking events. It has been proposed to use this methodology to identify metabasins "on the fly" during parallel microcanonical molecular dynamics simulations [183]. This model has been superimposed onto a network model (see section 5.5) of the PEL in order to simulate aging dynamics [184]. A combination of metadynamics [185] with the Markovian network model has been adapted to compute the viscosity of glasses, a feat which was previously impossible due to the long-timescale dynamics [186].

A related approach employs continuous time random walks (CTRW), which are differentiated from random walks by characterising each step by a variable waiting time $\tau$, during which the walker is stationary, followed by an instantaneous displacement $\Delta x$. It has been shown that such a scheme can be numerically derived for a glass-forming system [187]. The CTRW scheme requires coarse-graining in order to apply it to the analysis of molecular dynamics simulations for two reasons: firstly, the particles are constantly moving, rather than being stationary before each "jump;" thus it is necessary to carefully define what constitutes a jump. Secondly, a jump occurs over a finite time, rather than instantaneously as assumed by the model. Warren and Rottler demonstrated the viability of CTRW as a variation of the "cage" or "trap" model by parameterising it using ultra-long term MD simulations of a binary Lennard-Jones glass. They observe that the key feature is that trap states are uncorrelated, and the trap energy is chosen from an exponential distribution at each step [188, 189]. Helfferich et al. detail the implementation of such a scheme, adding the further constraints that particles may not loop back to their starting positions, or oscillate between two states [190, 191]. It should be noted that this coarse graining makes CTRW capable of describing the long-timescale $\beta$-relaxation (in the parlance of MCT) of the system, when a particle breaks out of its cage, but not the short-timescale $\alpha$ relaxations.

\subsection{Network topology}

The PEL can be described as a set of local minima connected by transition pathways, forming an undirected complex network [192]. Such networks are described by a variety of metrics, one of which is the degree distribution, i.e. the distribution of probabilities of a minimum (vertex, in the language of networks) having a certain number of connections (edges). Potential energy landscapes are found to have a power law degree distribution, a so-called "scale-free" network (a common motif in many fields that employ graph theory), and there is a strong link between the distribution of basin hypervolumes in the PEL and the fractal packing of Apollonian hyperspheres [193]. In contrast with a random network, which has an exponential degree distribution, the power-law tail means there is a non-trivial chance of finding a vertex with abnormally many edges, a "hub". This feature leads to the phenomenon of small-world networks: the geodesic between two points is shorter in scale free networks than for random networks, since hubs act as conduits. As an example, the degree distribution of several Lennard-Jones clusters is shown in figure 8, showing a power law degree distribution for the larger clusters. This feature is exploited in crystal structure prediction, where the hubs are generally associated with low energy configurations and metabasins. There is a strong correlation between the degree of a minimum and its potential energy (the global minimum is generally the most connected vertex) for LennardJones clusters. This can be explained by the large hyperarea surrounding lower minima, which means there is a higher probability of finding a transition pathway to them [192].

Beyond the degree distribution, there is a rich phenomenology of complex networks that allows many different analyses and many parameters that can potentially be used to describe PELs. One example is the "community" structure, or clustering of hubs within a landscape - the tendency of of local minima to aggregate in metabasins, and metabasins to form basins of metabasins. One such study identified varying degrees of community structure in LennardJones clusters [194]. One feature of scale free networks is preferential attachment: when the network grows upon adding more atoms and as a result more inherent 


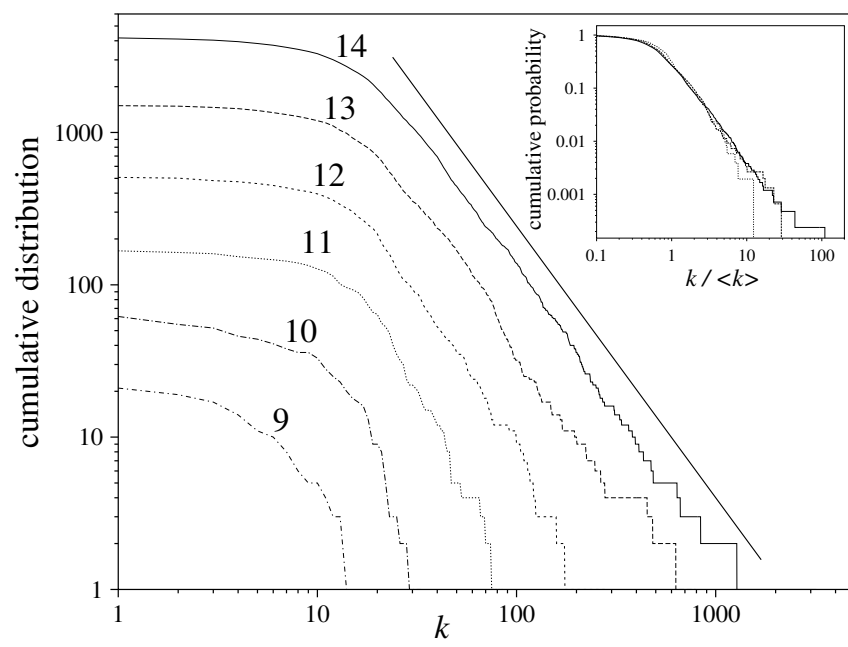

Figure 8. Cumulative degree distribution for Lennard-Jones clusters 9-14 (Reproduced with permission from J. Chem. Phys. 122, 84105 (C)2005, AIP Publishing LLC [192]). $k$ is the degree of a graph vertex, i.e. the number of connected edges. The graph gets progressively closer to a power law distribution with slope $-(\gamma-1)$, and $\gamma=2.78$ (straight line). Since the behaviour does not hold for the smaller clusters, the system is not truly scale free, but for larger clusters, there is a power law degree distribution.

states, the power law tail of the degree distribution arises from a tendency of new edges to be formed by preferentially attaching to verticies with a higher degree. It transpires that this behavior can be influenced by varying the range of the LennardJones potential by adjusting the exponents (which are usually 12 and 6). A shorter range potential (smaller exponents) is known to result in a more complex, rougher PEL with more vertices and edges in its network, since the weaker interaction from distant particles leads to a larger number of stable packings. The effect of increasing the range of the potential can be more rigorously explained using catastrophe theory [195]. Since the number of edges increases faster than the number of nodes, and these new edges undergo preferential attachment, leading to a scale free network [196].

Up to this point, the construction of the network has dispensed with all notions of activation barriers; however, it is possible to generalise this approach for a treatment of dynamics by, for example, superimposing a master equation onto the network [197, 198]. The problem with this approach is defining connectivity: in order to find connections between local minima, eigenvector-following algorithms are often employed in order to locate saddle points along the paths between basins with the lowest barrier. In this context, the definition of a connection between two minima is not well defined, and moreover, there is evidence to suggest that it is incorrect to use minima of the gradient of the total energy, since the majority of points where $|\nabla E|^{2}=0$ are non-stationary points of the potential energy [172]. In light of this finding, a different approach may be necessary.

In a related approach, Wang et al. [127] propose the "potential energy landscape ensemble", which is defined as the set of configurations for which the potential energy is less than or equal to some constant "landscape energy", $E_{L}$. This has the effect of deemphasising the concept of dynamics between basins as a process of surmounting activation barriers. In this picture, slow dynamics below the glass transition temperature is a result of the long and tortuous, but effectively barrierless, routes between basins i.e. the glass transition is dictated by entropic rather than energetic considerations (figure 9). This can be physically justified by the exponential dependence of barrier height on the probability of a barrier transition: crossing many low barriers is far more likely than crossing one high barrier.

In the case of an extremely low $E_{L}$, it is possible to envisage disconnected regions of the ensemble with no connected pathways, which corresponds to the transition to the non-ergodic regime. The extension that configurations follow geodesic paths between basins [128] may provide a more natural definition for network connectivity than the more common model of activated dynamics. In the case of the binary Lennard-Jones mixture, it has been shown that as the temperature decreases, geodesics increase as paths between minima become progressively more convoluted, much more so for glass-forming liquids [128]. Intriguingly, they observe the same behaviour (and successfully predict the onset of dynamic heterogeneity) for a hard sphere system, which has no inherent states or intervening barriers [199], raising the question of how much of the behaviour can be attributed to dynamics unique to a hard sphere system, which differs from more realistic soft potentials in some very important ways. The potential energy landscape ensemble paradigm has stimulated interest in NVU (constant potential energy) dynamics. Physically, NVU dynamics is a manifestation of Newton's second law: it models a system moving at constant velocity with zero friction, such that the force is always normal to the surface and thus does no work, conserving kinetic energy. It has been shown that NVU dynamics is equivalent to microcanonical (NVE) in the limit of large $N$ [200], and its results are as good as for canonical (NVT) and stochastic dynamics (such as Monte Carlo) [201].

\subsection{Discussion}

The general trend in the literature is to conclude that the glass transision can only be described in terms of non-local, collective rearrangement of atoms, rather 


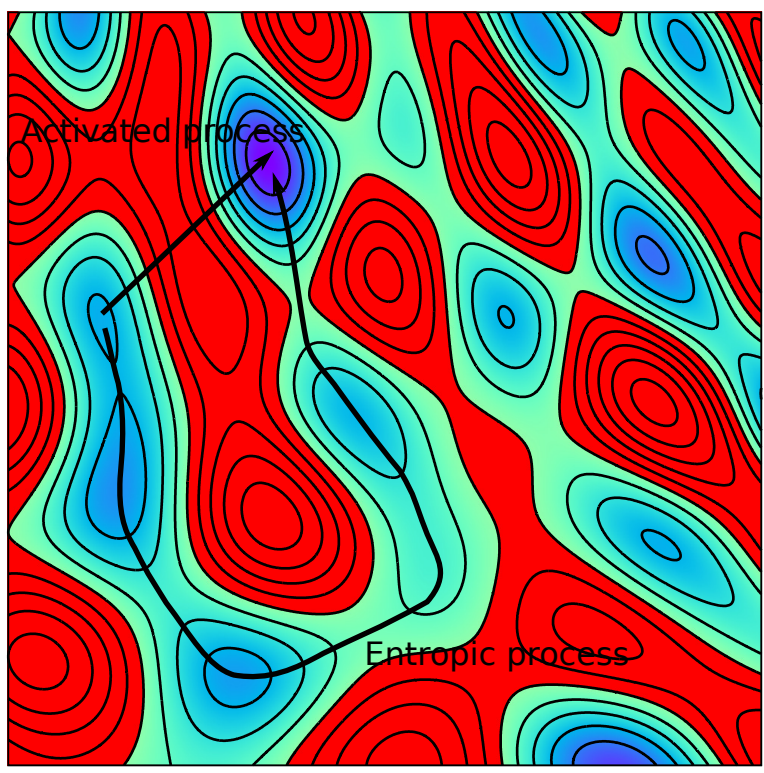

Figure 9. A two-dimensional illustration of the potential energy landscape ensemble (adapted from reference [128]). The ensemble consists of configurations with $V(\boldsymbol{R})<E_{L}$. As $E_{L}$ is decreased, the forbidden regions (red) become larger, and the paths between minima become longer and more tortuous, resulting in slow dynamics; the potential energy landscape problem essentially recasts an activated process as an entropic process.

than localised events or the static structure. There is, however, evidence to suggest that there are strong links between the potential energy landscape and dynamics, and that aspects of the dynamic heterogeneity arise from the static structure [147].

Single-structure descriptions of glasses do not in general tell the whole story. In terms of dynamical heterogeneity, free volume models of glasses are a generally unreliable descriptor, since free volume is a single particle property. Similarly, describing glasses as "disordered crystals" consisting of polyhedral units of the crystalline structure also fail when the local coordination in the liquid phase differs from the crystalline phase. Voronoi tesselation offers a useful, rigorous definition of free volume that demonstrates icosahedral short-range ordering in metallic glasses, where weak orientational constraints on the first coordination sphere turn the glass structure in to a hard sphere packing problem rather than a chemical bonding problem.

By introducing the PEL, we examine not only the static structure, but the whole ensemble of stable structures. A transition between two points on the PEL generally involves the movement of more than one particle, so such an approach is capable of describing collective motion. Just by looking at the structural density of states of randomly generated structures, it can be seen that glass-formers and crystal-formers have very different PELs: glass-formers are characterised by a low energy "long tail", that is, a high density of states close to the crystalline minimum. We can refine this picture by additionally considering either entropic or activated transition pathways between the local minima. Thus far, most analyses of this type on glassy systems have been primarily qualitative, so there is a need for more work treating network models of glasses quantitatively. One way of achieving this is by mapping minima and transition paths of a PEL to the vertices and edges of a complex network and performing statistical analysis on this simplified model. The converse can also be achieved, starting from a network model of a glass as a means of coarse-graining, and modelling a real-time trajectory between the local minima using a rate equation.

The dynamics of the system are implicit in the PEL. The dynamical matrix can be computed, which in turn can be used to determine the mechanical stability and properties of any configuration. Such methods may also be used to describe the the aging of glassy systems over timescales inaccessible to simulation using transition state theory, and unstable and diffusive modes offer an alternative definition of transition pathways. In such a way, modelling techniques that may be individually insufficient can be combined to offer a better understanding of glasses through a very simple paradigm, the PEL.

\section{Model glass-forming systems}

Ideally, we want to use fully transferable $a b$ initio potentials generated "on the fly" using density functional theory (DFT) [202, 203] to characterise the difference between good and bad glass formers. However, there is a wealth of research in the literature employing computationally cheap, idealised forcefield models to study potential energy landscapes. In this section, we summarise these results.

\subsection{The binary Lennard-Jones (BLJ) glass}

The binary Lennard-Jones mixture is one of the most commonly used model systems for glass formation, aptly described as "the "drosophila" of computational studies of glass-forming systems' [122]. Classical forcefields are computationally cheap, and allow molecular dynamics simulations on a timescale of milliseconds (compared with nanoseconds for $a b$ initio molecular dynamics). Moreover, a judicious choice of forcefield parameters and composition results in the tendency towards crystallisation to be suppressed. Many studies in the literature employ a potential similar to that of Kob and Andersen, a binary mixture of Lennard-Jones particles with the same mass in an 80:20 ratio [28]. The forcefield parameters were chosen 
to be similar to amorphous $\mathrm{Ni}_{80} \mathrm{P}_{20}$ in Weber and Stillinger's earlier model [204] to avoid crystallisation at low temperatures. Above its melting temperature, the BLJ is a fragile liquid and is good glass former. It is well characterised in the literature, with a known Kauzmann temperature [205], and it undergoes a second order phase transition to a glassy state at its mode-coupling temperature.

An explicit computation of the density of states of this system as a function of temperature reveals that the curve of logarithm of the density of states becomes steeper as the energy decreases [206]. This is not unexpected, and confirms that as the temperature decreases, the number of accessible states becomes smaller. Moreover, the number of pathways between minima decreases as the temperature decreases, an effect which is more pronounced for fragile systems, facilitating the super-Arrhenius behaviour of glasses. This can be linked to the cage picture, in which a particle is trapped in a cage formed by its nearest neighbours, such that a considerable barrier must be overcome for it to break out [207]. The selfdiffusion constant for individual atoms show Arrhenius behaviour over short timescales, and super-Arrhenius behaviour is recovered after averaging over all atoms over an ergodic timescale. This results not from the distribution of barriers, but from the negative correlation in atomic displacements over successive timesteps, i.e. reversals in direction, which may be analogous to non-productive cage-breaking (see section 5.4) [208, 209].

In the PEL picture, relaxations on two timescales are demonstrated by the BLJ mixture: high frequency $\alpha$-process, corresponding to transitions between minima within a metabasin, and a lowfrequency $\beta$-process, corresponding to transitions between metabasins. Above $T_{\mathrm{MCT}}$, the liquid is a multitrap system, for which the barriers separating inherent structures are small compared to the kinetic energy, and a single-trap system below it, when the barriers become large [210]; in this way it is possible to rigorously define metabasins in the context of a numerical simulation. Metabasins can be divided into liquid- and solid-like categories based on the "waiting time" $\tau$ of the configuration therein: solid-like metabasins have a waiting time $\tau>\tau *$, where $\tau *$ is a crossover value, and vice versa for liquid-like metabasins [211]. This is further evidence that the dynamical heterogeneity may have a static structural origin.

For small periodic BLJ systems such as the 32 atom system described in reference [83], or small BLJ clusters, it is possible to almost completely map the minima of the PEL; however, such systems are subject to finite size effects. The surface effects present in clusters are well known, but it has been shown that that finite size effects are insignificant for $N \geq$ 60 [212]. Since BLJ simulations are so cheap and can be productively performed on a modern desktop computer, it is tempting to examine larger systems, but there is a caveat: Doliwa and Heuer demonstrate that a 130 atom BLJ system essentially behaves as two weakly coupled 65-atom BLJ subsystems [213]. If the subsystems are sufficiently weakly correlated, it therefore makes sense to simulate only one subsystem to avoid obfuscating the information contained in the PEL [214]. Transitions between local minima are frequently modelled as a random walk, but this turns out not to be the case for hops between single basins; however hops between basins within a metabasin strongly resemble a random walk [215].

A simple thermodynamic model suggests that the fragility of a liquid is proportional to the logarithm of the number of states [216]. Sastry uses a BLJ mixture to establish a quantitative connection between fragility and the PEL [217]. In addition to the multiplicity of states (encoded in the configurational entropy), the fragility also depends on the variance of the distribution of states and the variation of vibrational frequencies (i.e. entropy) between the lowest and highest basins.

A phenomenological observation associated with the fragile BLJ liquid is the tendency for correlated rearrangements of clusters at temperatures above $T_{\mathrm{MCT}}$ which are accessible by molecular dynamics. Particle displacements appear to happen along stringlike clusters [118, 218]. In a glass, most atomic motion takes the form of small amplitude vibrations, but the structure is jammed on a diffusive timescale. A region of jammed molecules can only become mobile if it is adjacent to an unjammed region, a process which forms distinct chains [219].

Recently, a new molecular dynamics technique using a BLJ model was used to attempt to generate a model glass with the characteristics of ultrastable organic and bulk metallic glasses synthesised by vapour deposition. Groups of particles were introduced to a simulation cell some distance above a constrained substrate layer, followed by an equilibration phase and finally an energy minimisation. The resulting glass was found to have the properties of a vapour deposited equilibrium supercooled liquid, and it was noted that the optimal temperature for preparation of these ultrastable glasses corresponds to the Kauzmann temperature [10]. It is notable that the radial distribution function of this model ultrastable glass is indistinguishable from an ordinary quench glass; instead, the stability has been explained in terms of the cage picture. In a bulk glass, the motion of a particle is strongly constrained by the cage formed by its nearest neighbours; however, on the surface, it is free to "roll" 

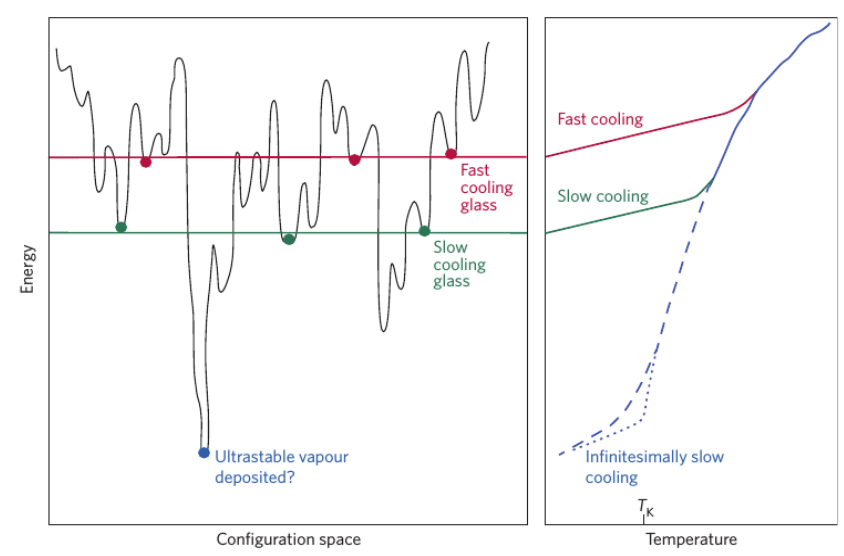

Figure 10. Schematic of the effects of cooling rate versus vapour deposition on glass stability (Reprinted by permission from Macmillan Publishers Ltd: Nature Mater. 12, 94, (C)2013 [220]). When a liquid is cooled, it falls out of equilibrium at a temperature that depends on the cooling rate. Lower cooling rates allow the system to explore lower energy metabasins, while vapour deposition and the molecular dynamics technique of Parisi et al. make extremely deep and narrow minima accessible.

with a reduced free energy cost, enhancing its mobility and allowing a more extensive exploration of the PEL [220] (figure 10). It should, however, be noted that this pciture is at odds with experiments suggesting that ultrastable metallic glasses are not necessarily the lowest thermodynamic state, but are kinetically very stable [9]. The question of whether ultrastable glasses are in fact the most thermodyanmically stable remains an open question in the literature.

As a final note, ring-polymer molecular dynamics (RPMD), which goes beyond Born-Oppenheimer molecular dynamics and simulates nuclei as quantum mechanical particles, has been used in conjunction with a BLJ mixture [221]. The degree of "quantumness" is controlled by the $\Lambda^{*}$ parameter, which is the ratio of the de Broglie wavelength to the particle size. Interestingly, the quantum mechanical glass is much less structured than the classical, since quantum mechanical tunneling across PEL barriers is permitted. Moreover, the diffusivity decreases as a function of $\Lambda^{*}$ until the size of the quantum wave packet becomes comparable to the radius of the cage enclosing the particle in the glass (at approximately $\Lambda^{*}=1$, i.e. a de Broglie wavelength comparable to the particle size), at which point it can tunnel through the cage, resulting in a sharp increase in diffusivity. Whilst this is a nice illustration of a theoretical quantum glass, this effect is unlikely to affect a real glass, for which the temperature regime will be too high, and the atoms too massive unless it contains hydrogen. More recent calculations suggest that quantum effects tend to broaden the glass transition regime [222].

\subsection{Amorphous silica}

Silica $\left(\mathrm{SiO}_{2}\right)$ is important not only because of its ubiquity in natural and man-made amorphous materials, but also because it is an extreme case of a strong liquid as can be seen by its position on the Angell plot (figure 1). Molecular dynamics simulations at varying pressures demonstrate that silica is a strong liquid at ambient pressure, when the silicon atoms are largely four-coordinated, but as the pressure increases, they become five- and six-coordinated, signalling the onset of fragility [223]; this was recently confirmed experimentally [224].

The availability of a reliable classical potential that has been validated against $a b$ initio models, the van Beest-Kramer-van Santen (BKS) potential [225], means it has been very well studied. A study on the PEL topology of BKS silica suggests a characteristic temperature $T_{c}=3500 \mathrm{~K}$, above which the system explores anharmonic basins, but below which there is no sign of anharmonicity [226]. Silica is also notable for its transition from a fragile liquid to a strong liquid as the temperature decreases below a crossover point, $T_{c}$, which is found to be around $3300 \mathrm{~K}$ [227] for the BKS model. This transition has been attributed to a low energy cutoff in the density of structural states, which coincides with the vanishing of the number of threeand five-coordination defects [228]. The similarity of the two aforementioned crossover temperatures suggest that there is a possible connection between basin anharmonicity and liquid fragility. $T_{c}$ coincides with a peak in the specific heat $C_{V}$, the maximum density and a maximum in the isothermal compressibility $K_{T}$, phenomena which are associated with a polyamorphic transition in glasses, i.e. the transition from a lowdensity glass to a high-density glass. This suggests that the fragile-strong transition is associated with a polyamorphic transition, and moreover that all strong liquids are candidates for polyamorphism [229]. This can be physically justified by the existence of two different arrangements of particles in tetrahedrally coordinated materials resulting in two characteristic interparticle distances. For example, low density ice Ih and the compact high pressure phases in the case of water [37], which also displays a fragilestrong liquid transition [230]. Kushima et al. [231] extend this to all viscous liquids, suggesting that there are two crossover temperatures for all glass formers: upon cooling from high temperature, there is a strong to fragile transition, followed by a fragile regime, and finally a fragile to strong transition. The difference between fragile and strong liquids lies in the rearrangement processes that occur between basins. Bond switching processes have high activation barriers prevalent at high temperatures, compared with processes involving dangling bonds which dominate at 
low temperatures. In the cage entrapment paradigm, high-barrier mechanisms correspond to cage-breaking, while low barrier mechanisms are related to the redistribution of free volume [207]. However, the barrier height is in general independent of temperature, except in a limited "intermediate" temperature range, over which the barrier changes with temperature, and the liquid is fragile [231].

\subsection{Hard sphere model}

A system of hard spheres is arguably the simplest system that displays fluid-solid coexistence and a glass transition. Hard spheres are essentially particles with short range repulsive interactions, and without longer range dispersive interactions; they do not interact unless they overlap. A hard sphere model places the emphasis on efficient packing rather than chemical bonding, and is often used to describe the glass transition as a "jamming" transition as in the case of granular materials, the point at which a system develops a yield stress in a disordered state [232].

This model has a single control parameter, the dimensionless density $n *=\rho_{0} \sigma^{3}$, where $\rho_{0}$ is the average density in the crystalline phase and $\sigma$ is the hard sphere diameter. Increasing $n *$ has the same effect as increasing the temperature [97]. A hard sphere system will remain structurally disordered if cooled fast enough, and under further compression it will jam into one of a multitude of possible packings with a minimal free volume, reaching the limit of "random close packing" at an infinite compression rate [233].

A study on devitrification (crystallisation from a glassy or amorphous phase) shows in order to form crystallites, particles undergo a short range "shuffling" motion, for which the mean displacement is less than a diameter - i.e. crystallisation occurs in spite of the arrest of diffusive motion [234]. The hard sphere free energy landscape undergoes a pressureinduced 'roughness transition', at which point basins become aggregates of sub-basins in a fractal manner (figure 11), representing a multitude of marginally stable states [235]. Expanding on these interesting results using more realistic models may eventually reveal the microscopic root of glass-forming ability in comparison with crystal-forming ability.

O'Hern et al. demonstrate that a hard sphere system undergoes a jamming transition, defined as the point at which the bulk and shear moduli simultaneously become non-zero, at a critical density, and proceed to show that this transition point has a variety of interesting properties, such as a diverging radial distribution function and an excess of lowfrequency normal modes. [232]. This supports the notion of cooperatively rearranging regions, since soft degrees of freedom are collective. Moreover, fewer and a
b
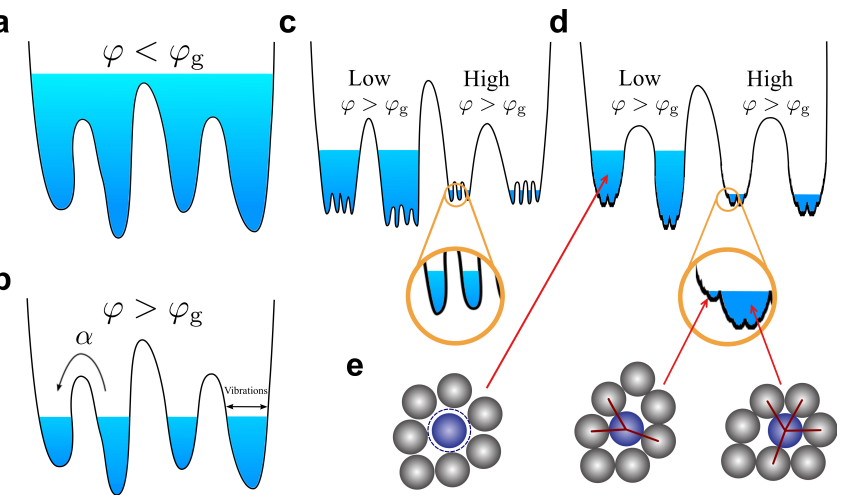

Figure 11. The pressure-induced transition to a fractal free energy landscape for hard spheres (Reprinted by permission from Macmillan Publishers Ltd: Nature Comm. 5, 3725 (C)2014 [235]). As the control parameter (packing fraction $\varphi$ ) is increased above its glass transition point $\varphi_{g}$, basins divide fractally into subbasins and sub-sub-basins representing marginally mechanically stable states close to a jamming transition.

fewer modes participate in structural rearrangement as the density approaches its critical value, perhaps suggesting cooperative regions that are growing in size [236].

At first glance, the hard sphere model appears to be somewhat academic, since real systems always have long range attractive forces. It does, however, remain a useful model, since all realistic systems have a distinct short-range repulsion cutoff, such as the minimum of a 6-12 Lennard-Jones potential. Moreover, at the density of solids, the primary effect of long-range attractions is to maintain the density of the solid such that the repulsions can have an effect [232]. Moreover, hard spheres are surprisingly good at explaining the glassforming ability of bulk metallic glasses, which are characterised by weak, non-directional interactions. In a binary hard sphere system, the best glass formers have an atomic size ratio that compromises between two competing pressures: minimising it to improve the packing efficiency, and maximising it to prevent phase separation [233].

A comprehensive review of hard sphere glasses can be found in reference [237].

\subsection{Discussion}

We have described model systems that describe very different types of glasses. On one hand, we have a binary Lennard-Jones model of amorphous silica, an example of a strong liquid that forms a glass with bonding network with high covalent character. At the other end of the spectrum, hard spheres are an idealisation of metallic glasses, derived from fragile liquids which lack the orientational constraints of strong liquids. The structure of such glasses becomes a question of filling space with spheres of different sizes, 
and a mechanical jamming transition, as opposed to the structure of a more rigid covalent bonding network.

The binary Lennard-Jones mixture is a good general choice of model system, since its glass-forming ability can be controlled via the composition, and its fragility can be controlled either by adjusting the hardness of the potential, or changing the particle density, which has essentially the same effect. Moreover, it is well characterised in the literature, and its mode-coupling temperature has been calculated for certain compositions and parameterisations.

An important point that arises from these cheap calculations is the effect of system size. One might naïvely assume that in order to capture medium-range ordering, it is necessary, due to the lack of symmetry, to use the largest supercell possible. This is not necessarily the case, as it has been demonstrating that once a critical system size is exceeded, the supercell can be decomposed in to weakly interacting subsystems, the effect of which may mask the properties of real interest.

\section{Ab initio models}

In addition to providing a more reliable and transferable structural model, ab initio methods are capable of describing electronic structure. Just as translational symmetry in crystals gives rise to Bloch electronic states which are delocalised, disorder in amorphous solids gives rise to localised states that can be more naturally described using Wannier functions [238]. Tight binding calculations on amorphous silicon manifest electronic states which appear as sparse but highly localised "islands" of electron density, and are caused by rare, major distortions in the local structure. These are often connected by "filaments" [239]. These structural filaments are related to the Urbach tail in amorphous silicon, the exponentially decaying density of states at the band edge [240, 241]. There is also a strong correlation between the degree of electronic localisation and the sensitivity of the electrons to lattice vibrations, i.e. strong localisation is associated with a larger electron-phonon coupling, as has been demonstrated for hydrogenated amorphous silicon [242, 243].

In fact, the paucity of $a b$ initio calculations on glasses reflects a general neglect of the effect of electronic structure on glass forming ability. There is evidence that glass forming ability is correlated with the electronic density of states. $\mathrm{Al}$ is a common component of bulk metallic glasses as a dopant, although it is rare to find $\mathrm{Al}$-rich $(>30 \%)$ glasses. It has been suggested that this is because $\mathrm{Al}$ has more free electrons (3) than most other metals, enhancing the density of states at the Fermi level and reducing the free energy of the amorphous state [244].
Icosahedral short range ordering is favoured by non-directional metallic bonds, but electronic structure calculations show that $\mathrm{Pd}_{40} \mathrm{Ni}_{40} \mathrm{P}_{20}$ achieves a superior packing ratio via a "hybrid" scheme involving both metal-centred icosahedra and highly directional covalently bound metalloid-centred tricapped trigonal prisms [245]. This is further evidence of the importance of electronic structure in glass-formation, since density functional theory is capable of transferably modelling glasses formed from both strong and fragile liquids.

A note of caution is in order when it comes to using local and semilocal DFT in the context of amorphous structures. When a defect is introduced into a crystal structure, it can introduce states into the band gap. These are manifested physically as localised states. Since glasses are very structurally disordered, there are many of these localised states, and in general local and semilocal exchange-correlation functionals will fail to capture the non-locality and correlations. This effect can be mitigated using hybrid exchangecorrelation functionals, but in the absence of any form of symmetry, these will be extremely expensive. One would expect to see qualitative as well as quantitative changes in the electronic structure as a result of this effect, which can have significant knock-on effects.

\section{Glass-forming ability versus crystal-forming ability}

Glass forming ability is a difficult quantity to characterise experimentally, since crystal nucleation depends on a variety of factors including temperature, pressure and composition, not to mention external factors such as chemical and physical impurities, container wall effects and shear flow condition in the liquid. To date, glass-forming ability has been described somewhat phenomenologically, for example, in the form of time-temperature-transformation curves (TTT) [246, 247]; other empirical formulae use the liquidus, crystallisation and glass transition temperatures to derive a parameter capable of predicting whether a bulk metallic glass has a high glass forming ability [13-15].

Experimentally, it has been possible to map the glass-forming ability of a 5-component alloy in composition space. Glass forming ability is controlled by two competing thermally activated processes: the Gibbs free energy barrier to the formation of a critical nucleus $W$ and the barrier to the configurational rearrangement of the liquid $\Delta G$. This can be summarised in the following expression for the characteristic nucleation time $\tau_{\alpha}$ of the crystalline phase $\alpha$ [248].

$$
\tau_{\alpha}=\eta^{-1} \exp \frac{1}{k T}\left[W(T, c)+\Delta G_{\alpha}(T, c)\right]
$$



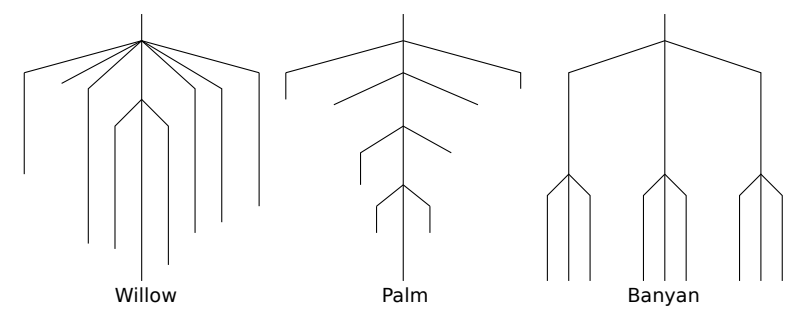

Figure 12. Disconnectivity graphs corresponding to three archetypal PELs (adapted from reference [251])

Here, the Gibbs free energy barriers $W$ and $\Delta G$ are functions of temperature and composition $(c)$, and $\eta$ is a transition attempt frequency.

Whilst there are many empirical guidelines for determining the glass-forming ability of compounds such as bulk metallic glasses, a microscopic rationale remains lacking [249]. Recent molecular dynamics simulations suggest a significant difference in the liquid-crystal interface between good and poor glass formers suggesting that a difference in heterogeneous susceptibilities may be responsible for the difference [250]. They can also be distinguished qualitatively by the "shape" of their respective PELs, although how to visualise such a high-dimensional space is a challenge in itself. Despa et al. achieve this by categorising disconnectivity graphs into three distinct motifs (figure 12): "weeping willow", in which the barriers are large compared with the relative energies of the basins, "palm tree", in which the barriers are small compared with the relative energies of the basins, and "banyan tree", which is characterised by many degenerate minima[251]. A glass former requires sufficiently high barriers below $T_{g}$, and a high degeneracy near the global minimum. In principle, the banyan and possibly willow tree motifs might be used to identify good glass formers.

The identification of compositions that would make good bulk metallic glasses used to be a time consuming trial and error procedure in which one composition could be generated per day. Recent developments have made the searching of composition space much more efficient [252], but gaps in the physical justification remain, and a theoretical method for generating glass forming compositions would streamline the process considerably.

\section{Conclusions and perspectives}

Over the course of this review, we have made the case that the PEL is a valid and useful paradigm for understanding glass formation. The dynamics of the system slow beyond any experimental, let alone computational timescale when the temperature is lowered to the point when glass transition intervenes and the system falls out of equilibrium. Thus stochastic sampling methods such as stochastic quenching and Monte Carlo are necessary to probe the energy landscape below the transition temperature.

Much of the work on energy landscapes has employed computationally cheap, and specialised or nontransferable models (in particular, binary LennardJones and hard sphere systems), limiting the predictive power of the PEL. Sampling using relatively expensive $a b$ initio techniques would facilitate theoretical predictions; calculations on this scale are certainly possible now, for periodic systems large enough to avoid finite size and small enough to avoid decoupling into non-interacting subsystems (around 60 atoms). This is true for atomic glasses, including bulk metallic glasses and amorphous thin films synthesised via vapour deposition, but perhaps not for more complex molecular, polymeric and organic glasses yet. These $a b$ initio models can in turn be used to generate coarse-grained models using activated dynamics and graph theory to further improve predictive power.

A criticism of PEL analysis is that it paints a largely static picture of glasses, and is incapable of describing the glass transition via, for example, a dynamic heterogeneity or a space-time phase transition. It can give us a wealth of information on the thermodynamically stable and metastable structure, and transitions between them, but can it tell us anything about the transition from a supercooled liquid to a glass? PEL analysis allows the development of structural models of glasses, but a static analysis is not likely to be sufficient to describe the glass transition - for example the static correlations are essentially identical between liquids and glasses, and no convincing connection has been made between diffusion and free volume in glass-forming liquids. However, there is evidence that the dynamic hetereogeneity may have a structural origin. One example is the correlation of the low frequency modes of a system with the propensity of particles to undergo large displacements; another is the observation of critical-like fluctuations in static structural order near the glass transition. Normal modes in particular are routinely derived from static structures via phonon calculations, and their application to more realistic models could be a fruitful area of research.

There are two key areas in which PEL analysis is likely to prove particularly useful: firstly in the generation of high quality structural models of glasses, and secondly in the identification of good glassforming compositions of metallic and vapour-deposited thin film glasses. The former can be achieved through some variation of stochastic quenching, and the latter through analysis of the density of states and network topology of the DFT energy landscape 
of real materials. At a fundamental level, it offers an attractive route to understanding the microscopic basis of glass formation.

\section{Acknowledgments}

This work was financially supported by the Swedish Foundation for Strategic Research (SSF) synergy program FUNCASE. Z. R. thanks Nuala M. Caffrey, Chris J. Pickard, Olle Hellman and Nina Shulumba for helpful discussions. B. A. acknowledges financial support from the Swedish Research Council (VR) grant No. 621-2011-4417. I. A. A. acknowledges support from SSF program SRL (Grant No. 10-0026, VR grant No. 621-2011-4426), the Russian Federation Ministry for Science and Education (grant No. 14.Y26.31.0005) and the Tomsk State University Academic D. I. Mendeleev funding program.

\section{References}

[1] Ding J, Cheng Y Q and Ma E 2014 Acta Mater. 69343

[2] Kaban I, Jóvári P, Kokotin V, Shuleshova O, Beuneu B, Saksl K, Mattern N, Eckert J and Greer A L 2013 Acta Mater. 612509

[3] Schuh C, Hufnagel T and Ramamurty U 2007 Acta Mater. 554067

[4] Schroers J 2013 Phys. Today 6632

[5] Swallen S F, Kearns K L, Mapes M K, Kim Y S, McMahon R J, Ediger M D, Wu T, Yu L and Satija S 2007 Science 315353

[6] Dawson K J, Kearns K L, Yu L, Steffen W and Ediger M D 2009 Proc. Natl. Acad. Sci. USA 106 15165

[7] Guo Y, Morozov A, Schneider D, Chung J W, Zhang C, Waldmann M, Yao N, Fytas G, Arnold C B and Priestley R D 2012 Nature Mater. 11 337

[8] Sepúlveda A, Tylinski M, Guiseppi-Elie A, Richert R and Ediger M 2014 Phys. Rev. Lett. 113045901

[9] Yu H, Luo Y and Samwer K 2013 Adv. Mater. 255904

[10] Singh S, Ediger M D and de Pablo J J 2013 Nature Mater. 12139

[11] Jansson U and Lewin E 2013 Thin Solid Films 5361

[12] Angell C A 1995 Science 2671924

[13] Ma D, Tan H, Wang D, Li Y and Ma E 2005 Appl. Phys. Lett. 86191906

[14] Mondal K and Murty B S 2005 J. Non-Cryst. Solids 3511366
[15] Du X H, Huang J C, Liu C T and Lu Z P 2007 J. Appl. Phys. 101086108

[16] Wang T, Gulbiten O, Wang R, Yang Z, Smith A, Luther-Davies B and Lucas P 2014 J. Phys. Chem. B 1181436

[17] Dyre J C 2006 Rev. Mod. Phys. 78953

[18] Johari G P and Goldstein M 1970 J. Chem. Phys. 532372

[19] Lifshitz I M, Gradescul S and Pastur L A 1988 Introduction to the theory of disordered systems (Wiley)

[20] Ruban A V and Abrikosov I A 2008 Rep. Prog. Phys. 71046501

[21] Stachurski Z H 2011 Materials 41564

[22] Ediger M D 2000 Annu. Rev. Phys. Chem. 5199

[23] Mendelev M I, Kramer M J, Ott R T, Sordelet D J, Besser M F, Kreyssig A, Goldman A I, Wessels V, Sahu K K, Kelton K F, Hyers R W, Canepari S and Rogers J R 2010 Philos. Mag. 90 3795

[24] Tanaka H, Kawasaki T, Shintani H and Watanabe K 2010 Nature Mater. 9324

[25] Faupel F, Frank W, Macht M, Mehrer H, Naundorf V, Rätzke K, Schober H R, Sharma S K and Teichler H 2003 Rev. Mod. Phys. 75237

[26] Kluge M and Schober H R 2001 Defect Diffus. Forum 194-199 849

[27] Buchenau U, Galperin Y M, Gurevich V L and Schober H R 1991 Phys. Rev. B 435039

[28] Kob W and Andersen H C 1995 Phys. Rev. E 51 4626

[29] Ediger M D and Harrowell P 2012 J. Chem. Phys. 137080901

[30] Kauzmann W 1948 Chem. Rev. 27219

[31] Sillescu H 1999 J. Non-Cryst. Solids 24381

[32] Middleton T F and Wales D J 2001 Phys. Rev. B 64024205

[33] Angell C A 1988 J. Phys. Chem. Solids 49873

[34] Wang L, Angell C A and Richert R $2006 \mathrm{~J}$. Chem. Phys. 125074505

[35] Yan L, Düring G and Wyart M 2013 Proc. Natl. Acad. Sci. USA 1106307

[36] Adam G and Gibbs J H 1965 J. Chem. Phys. 43 139

[37] Jagla E A 2001 Mol. Phys. 99753

[38] Mallamace F, Corsaro C, Leone N, Villari V, Micali N and Chen S H 2014 Sci. Rep. 43747

[39] Wei S, Yang F, Bednarcik J, Kaban I, Shuleshova O, Meyer A and Busch R 2013 Nat. Commun. 4 2083 
[40] Cahn J W and Bendersky L A 2003 An isotropic glass phase in al-fe-si formed by a first order transition Symposium MM Amorphous and Nanocrystalline Metals (MRS Proc. vol 806)

[41] Mendelev M I, Schmalian J, Wang C Z, Morris J R and Ho K M 2006 Phys. Rev. B 74104206

[42] Hedges L O, Jack R L, Garrahan J P and Chandler D 2009 Science $\mathbf{3 2 3} 1309$

[43] Angell C A 1991 J. Non-cryst. Solids 13113

[44] Chumakov A I, Monaco G, Monaco A, Crichton W A, Bosak A, Rüffer R, Meyer A, Kargl F, Comez L, Fioretto D, Giefers H, Roitsch S, Wortmann G, Manghnani M H, Hushur A, Williams Q, Balogh J, Parliski K, Jochym P and Piekarz P 2011 Phys. Rev. Lett. 106225501

[45] Stillinger F H 1990 Phys. Rev. B 412409

[46] Popova V A and Surovtsev N V 2014 Phys. Rev. E 90032308

[47] Fredrickson G H 1988 Ann. Rev. Phys. Chem. 39 149

[48] Stillinger F H 1995 Science $\mathbf{2 6 7} 1935$

[49] Berthier L and Biroli G 2011 Rev. Mod. Phys. 83 587

[50] Stillinger F H 1988 J. Chem. Phys. 887818

[51] Xia X and Wolynes P 2001 Phys. Rev. Lett. 86 5526

[52] Angell C A 1997 J. Res. Natl. Inst. Stand. Technol. 102102

[53] Tanaka H 2003 Phys. Rev. Lett. 90055701

[54] Johari G P 2002 J. Chem. Phys. 1162043

[55] Johari G P 2003 J. Chem. Phys. 25048

[56] Sciortino F, Kob W and Tartaglia P $2000 \mathrm{~J}$. Phys.: Condens. Matter 126525

[57] Gotze W 1999 J. Phys.: Condens. Matter 11 A1

[58] Das S P 2004 Rev. Mod. Phys 76785

[59] Reichman D R and Charbonneau P 2005 J. Stat. Mech. 2005 P05013

[60] Kirkpatrick T and Thirumalai D 2015 Rev. Mod. Phys. 87183

[61] Leutheusser E 1984 Phys. Rev. A 292765

[62] Bengtzelius U, Götze W and Sjölander A $1984 J$. Phys. C: Solid State Phys. 175915

[63] Barrat J L and Latz A 1990 J. Phys.: Condens. Matter 24289

[64] Nauroth M and Kob W 1997 Phys. Rev. E 55 657

[65] Foffi G, Götze W, Sciortino F, Tartaglia P and Voigtmann T 2003 Phys. Rev. Lett. 91085701

[66] Sciortino F and Kob W 2001 Phys. Rev. Lett. 86 648
[67] Bouchaud J P and Biroli G 2004 J. Chem. Phys. 1217347

[68] Lubchenko V and Wolynes P G 2007 Annu. Rev. Phys. Chem. 58235

[69] Xia X and Wolynes P G 2000 Proc. Natl. Acad. Sci. USA 972990

[70] Kirkpatrick T R and Wolynes P G 1987 Phys. Rev. A 353072

[71] Singh Y, Stoessel J P and Wolynes P G 1985 Phys. Rev. Lett. 541059

[72] Cavagna A, Grigera T and Verrocchio P 2007 Phys. Rev. Lett. 98187801

[73] Stevenson J D, Schmalian J and Wolynes P G 2006 Nature Phys. 2268

[74] Karmakar S, Dasgupta C and Sastry S 2009 Proc. Natl. Acad. Sci. USA 1063675

[75] Biroli G, Bouchaud J P, Cavagna A, Grigera T S and Verrocchio P 2008 Nature Phys. 4771

[76] Russell E V and Israeloff N E 2000 Nature 408 695

[77] Bhattacharyya S M, Bagchi B and Wolynes P G 2008 Proc. Natl. Acad. Sci. USA 10516077

[78] Debenedetti P G and Stillinger F H 2001 Nature 410259

[79] Stillinger F H and Weber T A 1982 Phys. Rev. A 25978

[80] Stillinger F 1999 Phys. Rev. E 5948

[81] Miller M A, Doye J P K and Wales D J $1999 \mathrm{~J}$. Chem. Phys. 110328

[82] Becker O M and Karplus M 1997 J. Chem. Phys. 1061495

[83] Heuer A 1997 Phys. Rev. Lett. 784051

[84] Pickard C J and Needs R J 2011 J. Phys.: Condens. Matter 23053201

[85] Ball K D, Berry R S, Kunz R E, Li F Y, Proykova A and Wales D J 1996 Science 271963

[86] De S, Schaefer B, Sadeghi A, Sicher M, Kanhere D G and Goedecker S 2014 Phys. Rev. Lett. 112 083401

[87] Sadeghi A, Ghasemi S A, Schaefer B, Mohr S, Lill M A and Goedecker S 2013 J. Chem. Phys. 139184118

[88] Wales D and Doye J P K 2001 Phys. Rev. B 63 214204

[89] Gommes C J, Jiao Y and Torquato S 2012 Phys. Rev. Lett. 108080601

[90] Doye J P K and Wales D J 1996 J. Chem. Phys. 1058428

[91] Bryngelson J D and Wolynes P G 1987 Proc. Natl. Acad. Sci. USA 847524 
[92] Bryngelson J D and Wolynes P G 1989 J. Phys. Chem. 936902

[93] Abraham S and Harrowell P 2012 J. Chem. Phys. 137014506

[94] Doye J P K, Miller M A and Wales D J $1999 \mathrm{~J}$. Chem. Phys. 1118417

[95] Doye J P K, Miller M A and Wales D J 1999 J. Chem. Phys. 1106896

[96] Wales D J and Bogdan T V 2006 J. Phys. Chem. $B 11020765$

[97] Dasgupta C and Valls O T 2000 J. Phys.: Condens. Matter 126553

[98] Jabbari-Farouji S, Wegdam G and Bonn D 2007 Phys. Rev. Lett. 99065701

[99] Odagaki T, Yoshidome T, Koyama A and Yoshimori A 2006 J. Non-Cryst. Solids 3524843

[100] Perera D N and Harrowell P 1998 Phys. Rev. Lett. 81120

[101] Perera D N and Harrowell P 2006 J. Non-Cryst. Solids 237314

[102] Yoshidome T, Yoshimori A and Odagaki T 2007 Phys. Rev. E $\mathbf{7 6} 021506$

[103] Goldstein M 1969 J. Chem. Phys. 513728

[104] Wallace D C 1997 Phys. Rev. E 564179

[105] Sastry S 2002 Phase Transit. 75507

[106] Sciortino F 2005 J. Stat. Mech. 200505015

[107] Sciortino F, Kob W and Tartaglia P 1999 Phys. Rev. Lett. 833214

[108] Sastry S 2000 Phys. Chem. Comm. 379

[109] McGreevy R L 2001 J. Phys.: Condens. Matter 13 R 877

[110] Biswas P, Tafen D N and Drabold D A 2005 Phys. Rev. B 71054204

[111] Zanotto E D 1998 Am. J. Phys. 66392

[112] Zhao J, Simon S L and McKenna G B 2013 Nat. Commun. 41783

[113] Hecksher T, Nielsen A I, Olsen N B and Dyre J C 2008 Nature Phys. 4737

[114] Elmatad Y Sand Chandler D and Garrahan J P 2009 J. Phys. Chem. B 1135563

[115] Elmatad Y S, Chandler D and Garrahan J P 2010 J. Phys. Chem. B 11417113

[116] Kob W and Barrat J L 1997 Phys. Rev. Lett. 78 4581

[117] Sastry S, Debenedetti P G and Stillinger F H 1998 Nature 393554

[118] Schrøder T B, Sastry S, Dyre J C and Glotzer S C 2000 J. Chem. Phys. 1129834
[119] Kob W, Sciortino F and Tartaglia P 2000 Europhys. Lett. 49590

[120] Saika-Voivod I and Sciortino F 2004 Phys. Rev. E $\mathbf{7 0} 041202$

[121] Diezemann G 2005 J. Chem. Phys. 123204510

[122] Lempesis N, Boulougouris G C and Theodorou D N 2013 J. Chem. Phys. 138 12A545

[123] Tsalikis D G, Lempesis N, Boulougouris G C and Theodorou D N 2008 J. Phys. Chem. B 112 10619

[124] La Nave E, Stanley H and Sciortino F 2002 Phys. Rev. Lett. $8 \mathbf{8} 035501$

[125] Angelani L, Di Leonardo R, Ruocco G, Scala A and Sciortino F 2000 Phys. Rev. Lett. 855356

[126] Broderix K, Bhattacharya K K, Cavagna A, Zippelius A and Giardina I 2000 Phys. Rev. Lett. 855360

[127] Wang C and Stratt R M 2007 J. Chem. Phys. 127224503

[128] Wang C and Stratt R M 2007 J. Chem. Phys. 127224504

[129] Ding K and Andersen H C 1986 Phys. Rev. B 34 6987

[130] Car R and Parrinello M 1988 Phys. Rev. Lett. 60 204

[131] Wang C Z, Ho K M and Chan C T 1993 Phys. Rev. Lett. 70611

[132] Biswas P, Tafen D N, Inam F, Cai B and Drabold D A 2009 J. Phys.: Condens. Matter 21084207

[133] Drabold D A and Li J 2001 Curr. Opin. Solid. St. M. 5509

[134] Yan Q, Jain T and de Pablo J 2004 Phys. Rev. Lett. 92235701

[135] Wooten F, Winer K and Weaire D 1985 Phys. Rev. Lett. 541392

[136] Barkema G T and Mousseau N 2000 Phys. Rev. $B \mathbf{6 2} 4985$

[137] Treacy M M J and Borisenko K B 2012 Science 335950

[138] Li N, Sakidja R, Aryal S and Ching W Y 2014 Phys. Chem. Chem. Phys. 161500

[139] Holmström E, Bock N, Peery T, Lizárraga R, De Lorenzi-Venneri G, Chisolm E and Wallace D 2009 Phys. Rev. E 80051111

[140] De Lorenzi-Venneri G and Wallace D C 2007 Phys. Rev. E $\mathbf{7 6} 041203$

[141] Kádas K, Andersson M, Holmström E, Wende H, Karis O, Urbonaite S, Butorin S M, Nikitenko S, Kvashnina K O, Jansson U and Eriksson O 2012 Acta Mater. 604720 
[142] Sykina K, Bureau B, Le Pollès L, Roiland C, Deschamps M, Pickard C J and Furet E 2014 Phys. Chem. Chem. Phys. 1617975

[143] Pickard C J and Needs R J 2006 Phys. Rev. Lett. 97045504

[144] Barkema G T and Mousseau N 1996 Phys. Rev. Lett. 774358

[145] Mousseau N and Barkema G T 2000 Phys. Rev. B 611898

[146] Rodney D and Schrøder T 2011 Eur. Phys. J. E 34100

[147] Widmer-Cooper A, Harrowell P and Fynewever H 2004 Phys. Rev. Lett. 93135701

[148] Gaskell P H 1978 Nature 276484

[149] Spaepen F 2000 Nature 408781

[150] Miracle D B, Sanders W S and Senkov O N 2003 Philos. Mag. 832409

[151] Sheng H W, Luo W K, Alamgir F M, Bai J M and Ma E 2006 Nature 439419

[152] Frank F C 1952 Proc. R. Soc. Lond. A 21543

[153] Doye J P K and Wales D J 1996 Science 271484

[154] Reichert H, Klein O, Dosch H, Denk M, Honkimäki V, Lippmann T and Reiter G 2000 Nature $\mathbf{4 0 8} 839$

[155] Luo W, Sheng H, Alamgir F, Bai J, He J and Ma E 2004 Phys. Rev. Lett. 92145502

[156] Xi X, Li L, Zhang B, Wang W and Wu Y 2007 Phys. Rev. Lett. 99095501

[157] Cheng Y, Ma E and Sheng H 2009 Phys. Rev. Lett. 102245501

[158] Fujita T, Konno K, Zhang W, Kumar V, Matsuura M, Inoue a, Sakurai T and Chen M 2009 Phys. Rev. Lett. 103075502

[159] Li Y, Guo Q, Kalb J A and Thompson C V 2008 Science 3221816

[160] Ma D, Stoica A D and Wang X L 2009 Nature Mater. 830

[161] Miracle D B 2004 Nature Mater. 3697

[162] Hirata A, Guan P, Fujita T, Hirotsu Y, Inoue A, Yavari A R, Sakurai T and Chen M 2011 Nature Mater. 1028

[163] Finney J L 1970 Proc. R. Soc. Lond. A 319479

[164] Borodin V A 1999 Philos. Mag. A 791887

[165] Voloshin V P, Naberukhin Y I, Medvedev N N and Jhon M S 1995 J. Chem. Phys. 1024981

[166] Starr F W, Sastry S, Douglas J F and Glotzer S C 2002 Phys. Rev. Lett. 89125501

[167] Widmer-Cooper A and Harrowell P 2006 J. NonCryst. Solids 3525098
[168] Cohen M H and Grest G S 1979 Phys. Rev. B 20 1077

[169] Calvo F, Bogdan T V, de Souza V K and Wales D J 2007 J. Chem. Phys. 127044508

[170] Derrida B 1981 Phys. Rev. B 242613

[171] Keyes T 2000 Phys. Rev. E 627905

[172] Doye J P K and Wales D J 2002 J. Chem. Phys. 1163777

[173] Heuer A 2008 J. Phys. Condens. Matter 20 373101

[174] Xu N, Frenkel D and Liu A J 2011 Phys. Rev. Lett. 106245502

[175] Widmer-Cooper A, Perry H, Harrowell P and Reichman D R 2008 Nature Phys. 4711

[176] Madan B and Keyes T 1993 J. Chem. Phys. 98 3342

[177] Zwanzig R 1983 J. Chem. Phys. 794507

[178] Keyes T 1994 J. Chem. Phys. 1015081

[179] Gezelter J D, Rabani E and Berne B J 1997 J. Chem. Phys. 1074618

[180] de Souza V K and Wales D J 2008 J. Chem. Phys. 129164507

[181] Monthus C and Bouchaud J P 1996 J. Phys. A: Math. Gen. 293847

[182] Tsalikis D G, Lempesis N, Boulougouris G C and Theodorou D N 2010 J. Phys. Chem. B 1147844

[183] Tsalikis D G, Lempesis N, Boulougouris G C and Theodorou D N 2010 J. Chem. Theor. Comput. 61307

[184] Boulougouris G C and Theodorou D N $2007 \mathrm{~J}$. Chem. Phys. 127084903

[185] Martoňák R, Laio A and Parrinello M 2003 Phys. Rev. Lett. 90075503

[186] Kushima A, Lin X, Li J, Eapen J, Mauro J C, Qian X, Diep P and Yip S 2009 J. Chem. Phys. 130224504

[187] Rubner O and Heuer A 2008 Phys. Rev. E 78 011504

[188] Warren M and Rottler J 2009 Europhys. Lett. 88 58005

[189] Warren M and Rottler J 2013 Phys. Rev. Lett. 110025501

[190] Helfferich J, Ziebert F, Frey S, Meyer H, Farago J, Blumen A and Baschnagel J 2014 Phys. Rev. E 89042603

[191] Helfferich J, Ziebert F, Frey S, Meyer H, Farago J, Blumen A and Baschnagel J 2014 Phys. Rev. E 89042604

[192] Doye J P K and Massen C P 2005 J. Chem. Phys. 12284105 
[193] Massen C P and Doye J P K 2007 Phys. Rev. E 75037101

[194] Massen C P and Doye J P K 2005 Phys. Rev. E 71046101

[195] Wales D J 2001 Science 2932067

[196] Massen C P and Doye J P K 2007 J. Chem. Phys. 127114306

[197] Kushima A, Eapen J, Li J, Yip S and Zhu T 2011 Euro. Phys. J. B 82271

[198] Banerjee S and Dasgupta C 2012 Phys. Rev. E 85021501

[199] Ma Q and Stratt R M 2014 Phys. Rev. E 90 042314

[200] Ingebrigtsen T S, Toxvaerd S, Heilmann O J, Schrø der T B and Dyre J C 2011 J. Chem. Phys. 135104101

[201] Ingebrigtsen T S, Toxvaerd S, Schrø der T B and Dyre J C 2011 J. Chem. Phys. 135104102

[202] Hohenberg P and Kohn W 1964 Phys. Rev. 136 B864

[203] Kohn W and Sham L 1965 Phys. Rev. 140 A1133

[204] Weber T A and Stillinger F H 1985 Phys. Rev. $B 311954$

[205] Coluzzi B, Parisi G and Verrocchio P $2000 \mathrm{~J}$. Chem. Phys. 1122933

[206] Faller R and de Pablo J J 2003 J. Chem. Phys. 1194405

[207] de Souza V K and Wales D J 2009 J. Chem. Phys. 130194508

[208] de Souza V and Wales D J 2006 Phys. Rev. B 74 134202 ISSN 1098-0121

[209] de Souza V and Wales D J 2006 Phys. Rev. Lett. 96057802

[210] Denny R, Reichman D and Bouchaud J P 2003 Phys. Rev. Lett. 90025503

[211] Doliwa B and Heuer A 2003 Phys. Rev. Lett. 91 235501

[212] Büchner S and Heuer A 1999 Phys. Rev. E 60 6507

[213] Doliwa B and Heuer A 2003 J. Phys.: Condens. Matter 15 S 849

[214] Büchner S and Heuer A 2000 Phys. Rev. Lett. 84 2168

[215] Doliwa B and Heuer A 2003 Phys. Rev. E 67 030501

[216] Speedy R J 1999 J. Phys. Chem. B 1034060

[217] Sastry S 2001 Nature 409164

[218] Donati C, Douglas J, Kob W, Plimpton S, Poole P and Glotzer S 1998 Phys. Rev. Lett. 802338
[219] Garrahan J P and Chandler D 2003 Proc. Natl. Acad. Sci. USA 1009710

[220] Parisi G and Sciortino F 2013 Nature Mater. 12 94

[221] Markland $\mathrm{T}$ E, Morrone J A, Berne B J, Miyazaki K, Rabani E and Reichman D R 2011 Nature Phys. 7134

[222] Novikov V and Sokolov A 2013 Phys. Rev. Lett. 110065701

[223] Barrat J L, Badro J and Gillet P 1997 Mol. Sim. 2017

[224] Zeidler A, Wezka K, Rowlands R F, Whittaker D A J, Salmon P S, Polidori A, Drewitt J W, Klotz S, Fischer H E, Wilding M C, Bull C L, Tucker M G and Wilson M 2014 Phys. Rev. Lett. 113135501

[225] van Beest B W H, Kramer G J and van Santen R A 1990 Phys. Rev. Lett. 641955

[226] Jund P and Jullien R 1999 Phys. Rev. Lett. 83 2210

[227] Horbach J and Kob W 1999 Phys. Rev. B 60 3169

[228] Saksaengwijit A, Reinisch J and Heuer A 2004 Phys. Rev. Lett. 93235701

[229] Saika-Voivod I, Poole P H and Sciortino F 2001 Nature 412514

[230] Angell C A, Bressell R D, Hemmati M, Sare E J and Tucker J C 2000 Phys. Chem. Chem. Phys. 21559

[231] Kushima A, Lin X, Li J, Qian X, Eapen J, Mauro J C, Diep P and Yip S 2009 J. Chem. Phys. 131 164505

[232] OHern C S, Silbert L E and Nagel S R 2003 Phys. Rev. E 68011306

[233] Zhang K, Smith W W, Wang M, Liu Y, Schroers J, Shattuck M D and O'Hern C S 2014 Phys. Rev. E 90032311

[234] Sanz E, Valeriani C, Zaccarelli E, Poon W C K, Pusey P N and Cates M E 2011 Phys. Rev. Lett. 106215701

[235] Charbonneau P, Kurchan J, Parisi G, Urbani P and Zamponi F 2014 Nat. Commun. 53725

[236] Brito C and Wyart M 2009 J. Chem. Phys. 131 024504

[237] Parisi G and Zamponi F 2010 Rev. Mod. Phys. 82789

[238] Drabold D A 2009 Euro. Phys. J. B 681

[239] Dong J and Drabold D A 1998 Phys. Rev. Lett. 801928

[240] Pan Y, Inam F, Zhang M and Drabold D 2008 Phys. Rev. Lett. 100206403 
[241] Drabold D A, Li Y, Cai B and Zhang M 2011 Phys. Rev. B 83045201

[242] Abtew T A, Zhang M and Drabold D A 2007 Phys. Rev. B $\mathbf{7 6} 045212$

[243] Drabold D A, Abtew T A, Inam F and Pan Y 2008 J. Non-Cryst. Solids 3542149

[244] Yu H B, Wang W H and Bai H Y 2010 Appl. Phys. Lett. 96081902

[245] Guan P F, Fujita T, Hirata a, Liu Y H and Chen M W 2012 Phys. Rev. Lett. 108175501

[246] Lu Z and Liu C 2003 Phys. Rev. Lett. 91115505

[247] Mukherjee S, Schroers J, Johnson W and Rhim W K 2005 Phys. Rev. Lett. 94245501

[248] Na J H, Demetriou M D, Floyd M, Hoff A, Garrett G R and Johnson W L 2014 Proc. Natl. Acad. Sci. USA 1119031

[249] Suryanarayana C, Seki I and Inoue A $2009 \mathrm{~J}$. Non-Cryst. Solids 355355

[250] Tang C and Harrowell P 2013 Nature Mater. 12 507

[251] Despa F, Wales D J and Berry R S 2005 J. Chem. Phys. 122024103

[252] Ding S, Liu Y, Li Y, Liu Z, Sohn S, Walker F J and Schroers J 2014 Nature Mater. 13494 